\title{
ONECUT2 is a driver of neuroendocrine prostate cancer
}

Haiyang Guo, Xinpei Ci ${ }^{2,3}$, Musaddeque Ahmed ${ }^{1}$, Junjie Tony Hua ${ }^{1,4}$, Fraser Soares ${ }^{1}$, Dong Lin ${ }^{2,3}$, Loredana Puca ${ }^{5}$, Aram Vosoughi ${ }^{5}$, Hui Xue ${ }^{2,3}$, Estelle Li ${ }^{2}$, Peiran Su ${ }^{1,4}$, Sujun Chen ${ }^{1,4}$, Tran Nguyen ${ }^{1}$, Yi Liang $^{1}$, Yuzhe Zhang 1,6,7, Xin Xu1, Jing Xu1, Anjali V. Sheahan ${ }^{8}$, Wail Ba-Alawi ${ }^{1,4}$, Si Zhang ${ }^{3}$, Osman Mahamud ${ }^{1,4}$, Ravi N. Vellanki ${ }^{1}$, Martin Gleave ${ }^{2}$, Robert G. Bristow (1) 1,4, Benjamin Haibe-Kains ${ }^{1,4,9,10,11}$, John T. Poirier (i) ${ }^{12}$, Charles M. Rudin (1) 12, Ming-Sound Tsao (1) 1,13, Bradly G. Wouters ${ }^{1,4,14}$, Ladan Fazli², Felix Y. Feng 15,16,17,18, Leigh Ellis (1) 8,19, Theo van der Kwast ${ }^{1,13}$, Alejandro Berlin (1) 1,14, Marianne Koritzinsky 1,14, Paul C. Boutros (1) 4,10,20, Amina Zoubeidi ${ }^{2}$, Himisha Beltran ${ }^{5}$, Yuzhuo Wang (1) ${ }^{2,3}$ \& Housheng Hansen He (i) ${ }^{1,4}$

Neuroendocrine prostate cancer (NEPC), a lethal form of the disease, is characterized by loss of androgen receptor (AR) signaling during neuroendocrine transdifferentiation, which results in resistance to AR-targeted therapy. Clinically, genomically and epigenetically, NEPC resembles other types of poorly differentiated neuroendocrine tumors (NETs). Through panNET analyses, we identified ONECUT2 as a candidate master transcriptional regulator of poorly differentiated NETs. ONECUT2 ectopic expression in prostate adenocarcinoma synergizes with hypoxia to suppress androgen signaling and induce neuroendocrine plasticity. ONEUCT2 drives tumor aggressiveness in NEPC, partially through regulating hypoxia signaling and tumor hypoxia. Specifically, ONECUT2 activates SMAD3, which regulates hypoxia signaling through modulating HIF1 $\alpha$ chromatin-binding, leading NEPC to exhibit higher degrees of hypoxia compared to prostate adenocarcinomas. Treatment with hypoxiaactivated prodrug TH-302 potently reduces NEPC tumor growth. Collectively, these results highlight the synergy between ONECUT2 and hypoxia in driving NEPC, and emphasize the potential of hypoxia-directed therapy for NEPC patients.

\footnotetext{
${ }^{1}$ Princess Margaret Cancer Centre, University Health Network, Toronto, M5G $1 \mathrm{L7}$ ON, Canada. ${ }^{2}$ The Vancouver Prostate Centre, Vancouver General Hospital and Department of Urologic Sciences, The University of British Columbia, Vancouver, V6H $3 Z 6$ BC, Canada. ${ }^{3}$ Department of Experimental Therapeutics, BC Cancer Research Centre, Vancouver, V5Z 1L3 BC, Canada. ${ }^{4}$ Department of Medical Biophysics, University of Toronto, Toronto, M5G 2M9 ON, Canada. ${ }^{5}$ Weill Cornell Medicine, New York, NY 10065, USA. ${ }^{6}$ College of Life Sciences, Central China Normal University, Wuhan, 430079 Hubei, People's Republic of China. ${ }^{7}$ College of Basic Medical Sciences, Dali University, Dali, 671000 Yunnan, People's Republic of China. ${ }^{8}$ Department of Oncologic Pathology, DanaFarber Cancer Institute, Boston, MA 02215, USA. ${ }^{9}$ Department of Computer Science, University of Toronto, Toronto, M5T 3A1 ON, Canada. ${ }^{10}$ Ontario Institute for Cancer Research, Toronto, M5G OA3 ON, Canada. ${ }^{11}$ Vector Institute, Toronto, M5G 1M1 ON, Canada. ${ }^{12}$ Memorial Sloan Kettering Cancer Center, New York, NY 10065, USA. ${ }^{13}$ Department of Pathology, University of Toronto, Toronto, M5G 1 L7 ON, Canada. ${ }^{14}$ Department of Radiation Oncology and Institute of Medical Science, University of Toronto, Toronto, M5T 1P5 ON, Canada. ${ }^{15}$ Department of Radiation Oncology, University of California at San Francisco, San Francisco, CA 94115, USA. ${ }^{16}$ Department of Urology, University of California at San Francisco, San Francisco, CA 94115, USA. ${ }^{17}$ Department of Medicine, University of California at San Francisco, San Francisco, CA 94115, USA. ${ }^{18}$ Helen Diller Family Comprehensive Cancer Center, University of California at San Francisco, San Francisco, CA 94115, USA. ${ }^{19}$ The Broad Institute, Cambridge, MA 02142, USA. ${ }^{20}$ Department of Pharmacology \& Toxicology, University of Toronto, Toronto, M5S 1A8 ON, Canada. These authors contributed equally: Haiyang Guo, Xinpei Ci. Correspondence and requests for materials should be addressed to H.G. (email: allen100006@gmail.com) or to Y.W. (email: ywang@bccrc.ca) or to H.H.H. (email: Hansenhe@uhnresearch.ca)
} 
$\mathrm{N}$ euroendocrine prostate cancer (NEPC) is a highly aggressive form of prostate cancer (PCa). Although it rarely arises de novo, NEPC may emerge from prostate adenocarcinoma (adeno-PCa) due to lineage plasticity induced by androgen receptor (AR)-targeted therapy ${ }^{1,2}$. A hallmark of NEPC is the loss of androgen receptor (AR) signaling during neuroendocrine transdifferentiation, resulting in resistance to ARtargeted therapy ${ }^{3}$. With the introduction of highly potent AR-targeted agents into the clinic, such as enzalutamide, the incidence of treatment-emergent NEPC ( $t$-NEPC) is expected to escalate $^{4,5}$. It has been reported recently that nearly one-fifth of metastatic CRPC develop small-cell neuroendocrine pathologic features after potent AR pathway inhibitor treatment ${ }^{6}$. Patients with NEPC have very limited therapeutic options, and the median overall survival is $<1$ year from time of diagnosis ${ }^{7,8}$. Understanding the molecular mechanisms driving t-NEPC is critical for improving therapeutic interventions for PCa patients.

NEPC resembles other poorly differentiated neuroendocrine tumors (NETs) such as small-cell lung cancer (SCLC), in both genomic and epigenetic alterations ${ }^{1,9,10}$. NETs, characterized by the presence of secretory granules and the production of hormones and amines ${ }^{11,12}$, are divided into well- and poorly differentiated subgroups based on their differentiation and proliferative index ${ }^{1}$. Well-differentiated NETs, which tend to be slow growing, show a pattern of trabecular, nested, or gyriform tumor cells with abundant neurosecretory granules ${ }^{13,14}$. In contrast, poorly differentiated NETs, which are more proliferative and aggressive, have diffuse or sheet-like architecture, abnormal nuclei and less cytoplasmic granularity ${ }^{13,14}$.

There are limited numbers of therapeutic options for patients with poorly differentiated NETs. Poorly differentiated NETs such as SCLC are typically treated with chemotherapy ${ }^{15}$. Most available systemic treatments are efficacious for only a limited time prior to the emergence of resistance. There is thus a great need for novel agents to improve remission rates and prolong overall survival. Understanding the common oncogenic mechanisms of poorly differentiated NETs could help identify common vulnerabilities across these aggressive tumors. Although a few pan-NET biomarkers have been identified, including chromogranin A and synaptophysin $^{11}$, the master regulators that are critical in driving poorly differentiated NET have not been systematically assessed. Since a single transcription factor (TF) can modulate multiple downstream pathogenesis-related genes, identification of master TFs of NETs could pinpoint more efficacious therapeutic targets for NETs.

To fill this knowledge gap, we performed a pan-cancer analysis of poorly differentiated NET and non-NETs, and identified transcription factor ONECUT2 as a potential master regulator of poorly differentiated NETs. ONECUT2 is significantly upregulated in NEPC compared to adeno-PCa. Ectopic expression of ONECUT2 drives NE plasticity and induces hypoxia response genes in PCa cells. Importantly, NEPC tend to be more hypoxic than adeno-PCa, suggesting that hypoxia-directed therapy may benefit patients with NEPC.

\section{Results}

ONECUT2 is a potential master transcriptional regulator of poorly differentiated neuroendocrine tumors. To identify potential master transcriptional regulators in NEPC, we performed differential mRNA abundance analysis in two independent castration-resistant prostate adenocarcinoma (adeno-CRPC) and NEPC transcriptome data sets ${ }^{10,16}$ (Fig. 1a). We identified 434 genes that are commonly upregulated in NEPC relative to adeno-CRPC (Supplementary Figure 1a). Considering the molecular similarity between NEPC and other types of poorly differentiated NETs ${ }^{1,9,10}$, we hypothesized that there are common master transcriptional regulators of poorly differentiated NETs. To test this hypothesis, we expanded our mRNA abundance analyses to two other cancer types, lung (i.e., SCLC and lung adenocarcinoma) and nervous system tumors (i.e., poorly differentiated neuroblastoma and glioma) using RNA-Seq data from Cancer Cell Line Encyclopedia (CCLE). With the same criteria, we identified 927 and 906 genes that are upregulated in the comparisons of poorly differentiated NETs vs. non-NETs in lung and nervous system cancer, respectively (Supplementary Figure 1a). Altogether, we identified a total of 88 genes that are upregulated in NETs vs. non-NETs in all three different cancer types, including 9 TFs (Supplementary Figures 1a-b and Supplementary Table 1). Importantly, well-characterized NET biomarkers like CHGA, SYP and SYT4 $4^{11}$, were identified in our 88-gene list, confirming the accuracy of our analysis pipeline. Three of the 9 TFs (i.e., ASCL1, INSM1, and PROX1) have been reported to be either important regulators or biomarkers of NETs ${ }^{17-19}$, while three others (i.e., SIX2, MYT1 and MYT1L) are functional in neuronal development or endocrine tissues ${ }^{20-22}$.

To explore the potential regulatory relationships between the 9 TFs and the 79 non-TF genes, we performed a modulatory analysis using a co-expression network based on mRNA abundance correlation in the three NE tumor types (see Methods). The top community comprised $60 \%$ of genes, including five TFs (MYT1, MYT1L, ZNF711, ONECUT2, and INSM1) with top weighted degrees (Fig. 1b). These five TFs comprised the majority of nodes in this network, suggesting that they are more likely to be crucial NET transcriptional regulators.

To evaluate the potential role of these five TFs in tumorigenesis, we analyzed their mRNA expression in tumor and normal samples from 20 cancer types in TCGA dataset. ONECUT2 was the most commonly upregulated TF, showing increased mRNA levels relative to normal in 15 cancer types (Fig. 1c). ONECUT2 is a member of the onecut family of transcription factors, known to be an important regulator of early retinal cell fate during development ${ }^{23}$. ONECUT2 modulates the expression of oncogenic lncRNA PCAT1 in prostate cancer, indicating its potential role in prostate cancer development ${ }^{24}$. However, its function in poorly differentiated NETs and NEPC is unknown.

ONECUT2 expression increases during PCa progression. ONECUT2 expression is significantly higher in human tumor samples of SCLC compared with lung adenocarcinoma (Supplementary Figure 2a), and in poorly differentiated neuroblastoma compared with glioma (Supplementary Figure 2b), consistent with the observation in CCLE cell line data. ONECUT2 expression is also elevated in a NEPC patient-derived xenograft (PDX) model LTL331R, which was developed from a hormone-naive prostate adenocarcinoma PDX model LTL331 through castration (Supplementary Figure 2c).

Besides the human NETs and non-NETs, we also analyzed Onecut 2 expression in genetically engineered mouse models of PCa. PCa tumors initiated in Pten and Rbl double knockout (DKO) model showed increased lineage plasticity and features of NEPC compared with Pten knockout and single copy Rb1 deletion (SKO) or normal prostate tissues (WT) ${ }^{25}$. Onecut2 expression was significantly elevated in DKO compared with SKO and WT (Supplementary Figure 2d), consistent with the observation of ONECUT2 upregulation in human NETs.

To further confirm its clinical relevance, we assessed ONECUT2 expression from benign prostate tissues, primary adenocarcinoma and metastatic CRPC tumors in two independent clinical cohorts and a pooled cohort of TCGA and Beltran ${ }^{10}$. We determined that ONECUT2 expression is significantly increased 
a

NET vs. non-NET

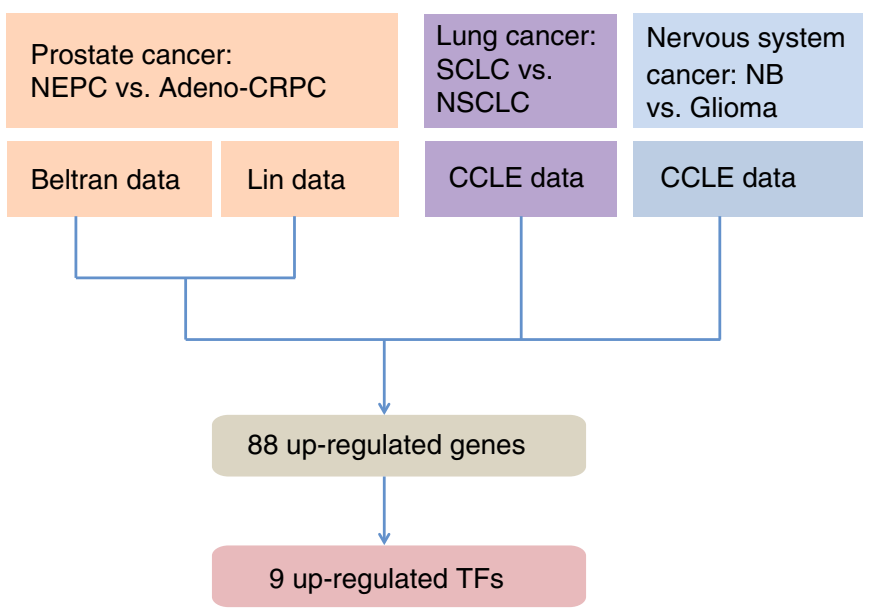

C 5 pan-NET up regulated TFs in TCGA (tumor vs. normal)

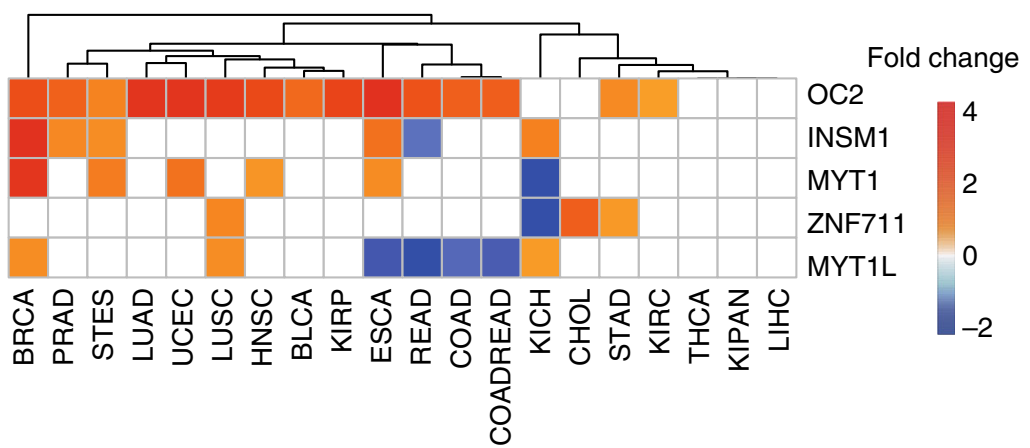

b

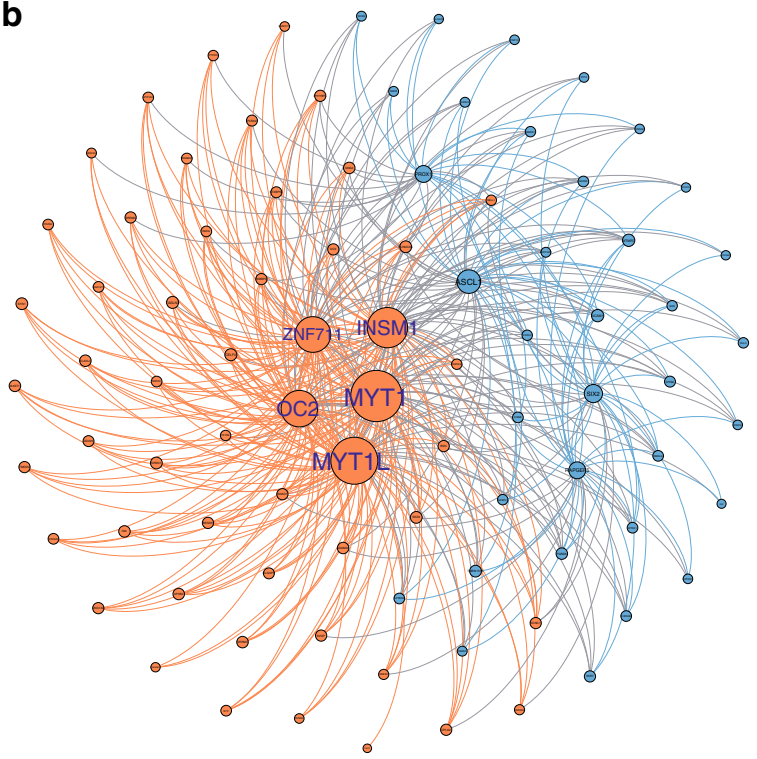

d

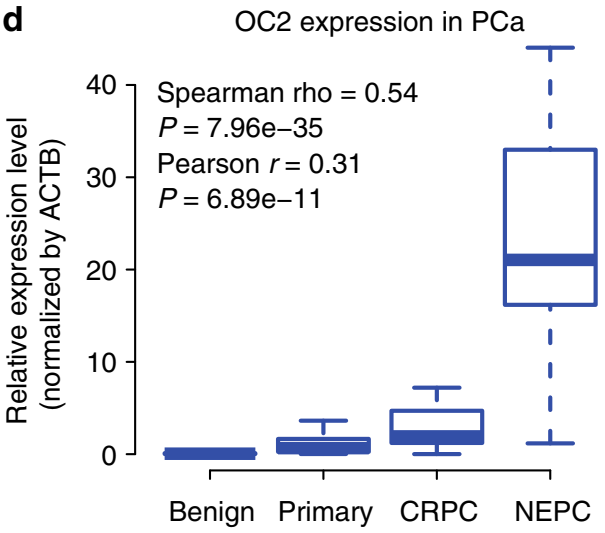

Fig. 1 Pan-cancer analysis identifies ONECUT2 as a potential master transcriptional regulator of neuroendocrine tumors. a Schematic illustration of panNET analysis (see Methods section and Supplementary Figure 1a for details). Ninety-three genes, including nine transcription factors, were commonly upregulated in NETs compared to the non-NET counterparts within the same cancer types. NET neuroendocrine tumor, NEPC neuroendocrine prostate cancer, Adeno-CRPC castration-resistant prostatic adenocarcinoma, SCLC small-cell lung cancer, NSCLC non-small-cell lung cancer (exclusive of large cell lung cancer), NB neuroblastoma, CCLE Cancer Cell Line Encyclopedia. b Network analysis of nine TFs and 79 non-TF genes commonly upregulated in poorly differentiated NETs. These 88 genes were classified into two communities using GEPHI, as labeled by color (orange and cyan). c Dysregulation of the five TFs from the major community from panel $\mathbf{b}$ in 20 cancer types from TCGA. $\log _{2}$ transformed fold changes represent difference in expression between tumor and normal tissue. Colored cells indicate significant expression changes (absolute-value of the $\log _{2}$ (fold change) $>1$ and $P<0.05$ ). The Wilcoxon rank sum test was used to calculate $P$-values. d ONECUT2 expression in benign prostate tissues and prostate tumors. RNA-Seq data of 52 benign prostate tissues and 333 primary PCa were retrieved from TCGA. RNA-Seq data of 34 adeno-CRPC and 15 NEPC were retrieved from the Beltran dataset. Expression levels as measured by RPKM were normalized by housekeeping gene ACTB

in CRPC compared to primary adenocarcinoma (Supplementary Figure 3a), while the largest difference is observed between NEPC and adeno-CRPC (Fig. 1d). Additionally, high level of ONECUT2 in primary adenocarcinoma is a strong predictor of poor outcome as measured by biochemical recurrence-free survival in two clinical cohorts (Supplementary Figure 3b). Collectively, these data suggest that ONECUT2 is associated with the progression of PCa.

ONECUT2 regulates hypoxia signaling in NE-like PC3 cells. To determine the function of ONECUT2 in NEPC, we silenced it in PC3 cells, an AR-negative PCa cell line with characteristics of prostatic small cell NE carcinoma ${ }^{26}$. ONECUT2 knockdown using two different siRNAs significantly decreased proliferation of NE-like PC3 cells (Supplementary Figure 4a-c). Consistently, silencing ONECUT2 using two different shRNAs significantly reduced subcutaneous tumor growth in PC3 xenografts (Fig. 2a; Supplementary Figure 4d). Similar effects on cell proliferation and tumor growth were observed in an additional NEPC cell line NCI-H660 and its cell line-derived xenograft models (Supplementary Figure 4e-f).

To further delineate the mechanisms underlying the function of ONECUT2 in NEPC, we performed RNA-Seq in PC3 cells with and without siRNA silencing of ONECUT2. We identified 67 and 93 genes significantly upregulated and down-regulated by ONECUT2, respectively (Supplementary Figure 5a). Gene Set Enrichment Analysis (GSEA) revealed that ONECUT2 targets genes were enriched with cell cycle related gene sets such as G2M_CHECKPOINT and E2F_TARGETS (Supplementary Figure $5 b$ ), in concordance with the function of ONECUT2 in regulating PC3 cell proliferation and tumor growth (Fig. 2a and Supplementary Figure 4c). Interestingly, we found enrichment of ANGIOGENESIS and HYPOXIA pathways in ONECUT2 
a

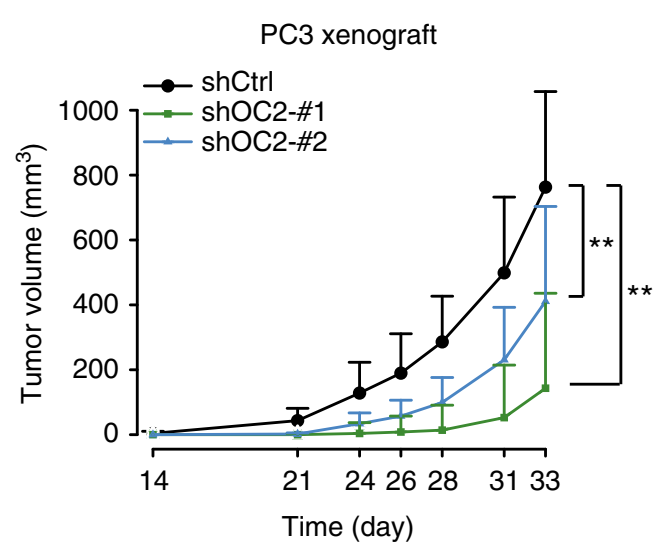

b

PC3 cells under hypoxic condition

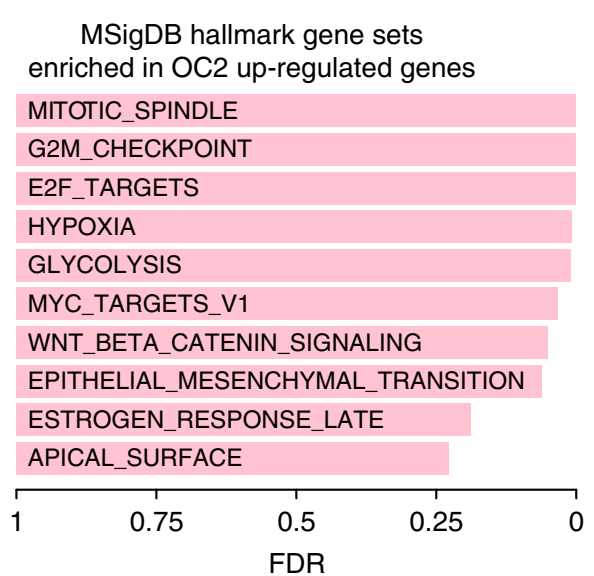

Hypoxia hallmark genes siCtrl siOC2

Enrichment plot: HALLMARK_HYPOXIA
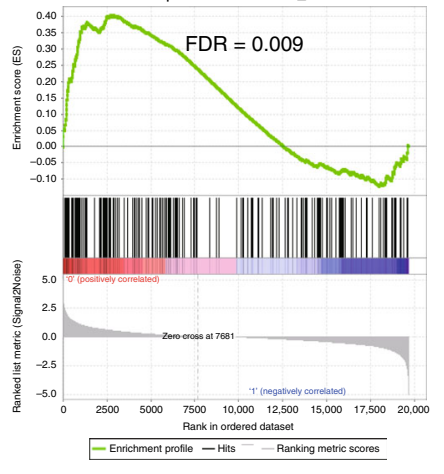

C

120 hypoxia-induced genes in PC3 cells

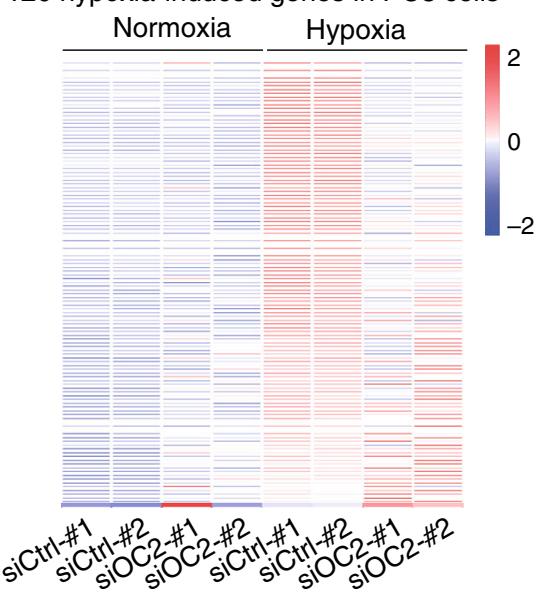

\section{d}

Hypoxia-induced genes in PC3 cells siCtrl siOC2

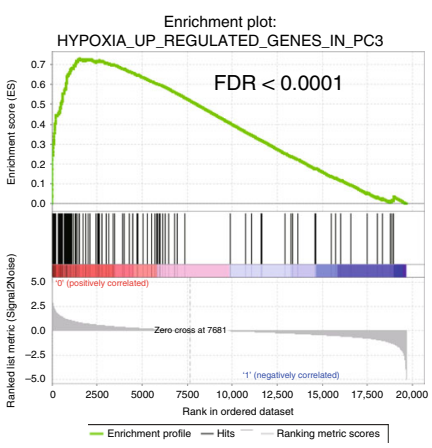

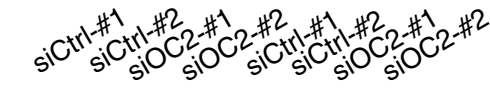

g PC3 cell migration

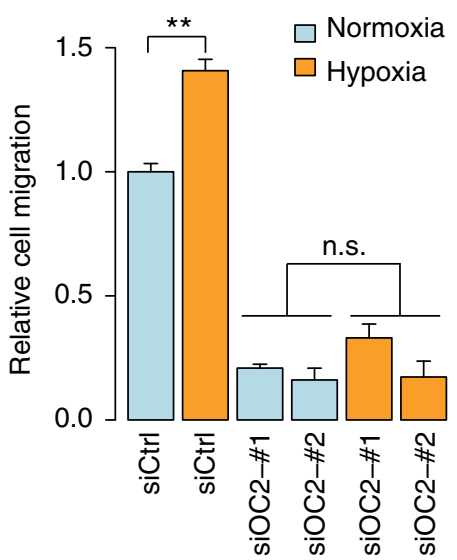

e

OC2-dependent hypoxia-induced genes

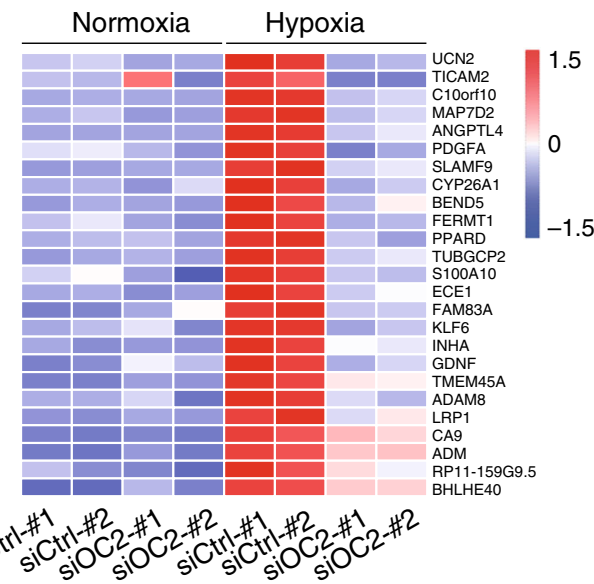

h

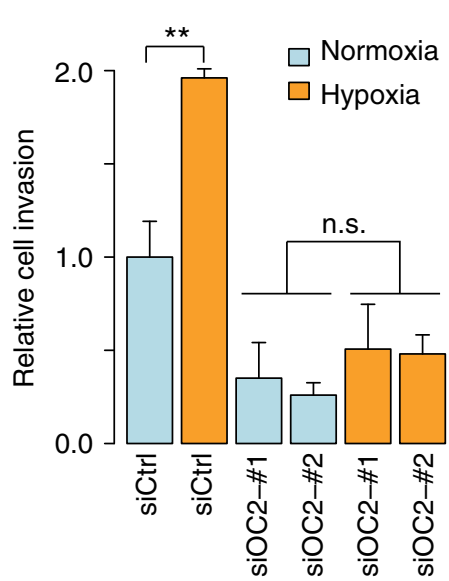

Fig. 2 ONECUT2 regulates hypoxia signaling in NE-like PC3 cells. a Lentiviral knockdown of ONECUT2 suppresses tumor growth in PC3 xenograft models. $n=9$ for shCtrl; $n=10$ for shOC2-\#1; $n=10$ for shOC2-\#2. b Left panel: top 10 MSigDB Hallmark Gene Sets enriched in ONECUT2 upregulated genes under hypoxic conditions. Right panel: GSEA enrichment plot for "Hypoxia" gene set. Genes were ranked by fold changes between control and ONECUT2 knockdown samples in descending order. c Heatmap shows relative expression of 120 hypoxia-induced genes (fold change $>2$ and $P$-value $<0.05$ ) in PC 3 cells with and without ONECUT2 knockdown under normoxic and hypoxic conditions. d GSEA enrichment plot of hypoxia-induced genes in PC 3 cells. Genes were ranked by fold changes between control and ONECUT2 knockdown samples in descending order. e Heatmap shows abundance of ONECUT2dependent hypoxia-induced genes. $\mathbf{f}$ Representative images of Pimonidazole (PIMO) IHC staining of PC3 xenograft tumors with and without silencing of ONECUT2. $\mathbf{g}$, $\mathbf{h}$ The capacity of PC3 cell migration and invasion with and without silencing of ONECUT2, under normoxic and hypoxic conditions in PC3 cells. $P$-values were calculated by mixed-effects models of repeated-measures ANOVA for $\mathbf{a}$, Wilcoxon rank sum test for $\mathbf{c}$ and one-way ANOVA for $\mathbf{g}$ and h. n.s. not significant; ${ }^{\star}: P<0.05 ;{ }^{\star \star}: P<0.01$. Source data are provided as a Source Data file 
regulated genes (Supplementary Figure 5b-c). Since hypoxia is strongly correlated with $\mathrm{PCa}$ progression and induction of NE plasticity in $\mathrm{PCa}^{27,28}$, we hypothesized that the function of ONECUT2 in NEPC is closely related with the regulation of cellular response to hypoxia.

To test this hypothesis, we analyzed ONECUT2 target genes in PC3 cells under hypoxic conditions using the ONECUT2 siRNA and RNA-Seq analysis as described above. GSEA identified very similar cell cycle related gene sets as with cells under normoxic conditions, while two hypoxia-related gene sets, HYPOXIA and GLYCOLYSIS, were markedly enriched in ONECUT2 upregulated genes under hypoxic conditions (Fig. 2b; Supplementary Figure 5d-e).

To identify hypoxia-responsive genes in PC3 cells, we profiled their transcriptomes under normoxic and hypoxic conditions using RNA-Seq. We identified 120 genes significantly induced by hypoxia (Fig. 2c). Interestingly, while only a small number of genes, such as CXCR4 and ARRDC3, tend to be modestly downregulated by ONECUT2 under both normoxic and hypoxic conditions, most of the 120 genes upregulated by ONECUT2 are specific under hypoxic conditions (Fig. 2c, d). Amongst the 120 genes, 25 were significantly upregulated by ONECUT2 under hypoxic conditions, including classical HIF-driven genes like $A D M$ and ANGPTL4 (Fig. 2e). These results suggest that ONECUT2 regulates hypoxia-induced gene expression in NEPC cells.

To determine the effect of ONECUT2 on tumor hypoxia, we analyzed hypoxia levels using pimonidazole (PIMO) staining in PC3 and NCI-H660 xenografts with and without silencing of ONECUT2. While tumors with control shRNAs were highly hypoxic, the hypoxia levels in ONECUT2 knockdown tumors were dramatically reduced (Fig. 2f; Supplementary Figure 6a-b). Silencing of ONECUT2 also significantly decreased PC3 cell proliferation under hypoxic conditions (Supplementary Figure $6 \mathrm{c}-\mathrm{d}$ ). Interestingly, while hypoxia treatment promoted PC3 cell migration and invasion, this effect was abrogated by ONECUT2 knockdown (Fig. 2g, h; Supplementary Figure 6e). These data suggest that ONECUT2 regulates tumor hypoxia and hypoxia-induced aggressive tumor biology in NEPC cells.

ONECUT2 regulates HIF1a binding to the chromatin. Since HIFla plays a central role in transcriptional response to hypoxia ${ }^{29,30}$ and ONECUT2 regulates hypoxia signaling and tumor hypoxia, we next sought to characterize the interplay between HIF1a and ONECUT2 at transcriptional regulation. ONECUT2 knockdown did not affect HIF1a protein levels under hypoxic conditions, and vice versa (Fig. 3a; Supplementary Figure 7a). However, HIFla genomic occupancy was globally decreased upon ONECUT2 knockdown as determined by ChIPseq analyses (Fig. 3b), which was exemplified by binding sites near ANGPTL4 and ADM (Supplementary Figure $7 \mathrm{~b}$ ). In addition, HIF1 $\alpha$ binding sites were strongly enriched near (within 20 $\mathrm{kb}$ of transcription start sites (TSSs)) ONECUT2 upregulated genes (Supplementary Figure 7c). Consistent with this, RT-qPCR analysis demonstrated that the induction of ANGPTL4 and ADM expression by hypoxia was significantly reduced by ONECUT2 knockdown (Fig. 3c). These data collectively suggest that ONECUT2 regulates hypoxia signaling through the regulation of HIFla binding to the chromatin.

ONECUT2 mediates HIF1a genomic binding through activation of SMAD3. We then explored the potential mechanism of how ONECUT2 regulates HIF1a chromatin binding in hypoxia response. Since ONECUT2 did not modulate HIF1a protein level, we compared ONECUT2 and HIF1a genomic binding sites (cistromes) in PC3 cell under hypoxic conditions. Only $8.4 \%$ of ONECUT2 and $7.6 \%$ of HIF1 $\alpha$ binding sites overlapped with one another (Supplementary Figure $7 \mathrm{~d}$ ), which cannot explain the genome-wide reduction of HIF1a binding after ONECUT2 knockdown (Fig. 3b). Consistently, ONECUT2 or HIF1a immunoprecipitation followed by HIF1a or ONECUT2 western blot analyses did not detect interaction between ONECUT2 and HIF1a. We thus further tested whether ONECUT2 would activate HIFla co-factors that modulate HIFla binding. We first identified 58 ONECUT2 direct upregulated genes that fulfill two criteria: (1) significantly upregulated by ONECUT2 in PC3 cells under hypoxic conditions; (2) have ONECUT2 bindings within $20 \mathrm{~kb}$ from their TSSs. This gene list was then filtered by motifs enriched in HIF1a binding sites in control PC3 cells compared with ONECUT2 knockdown cells (Supplementary Table 2). Further filtering with HIF1a interacting proteins pinpointed SMAD3 as a candidate HIF1a co-factor activated by ONECUT2 (Fig. 3d).

We identified a ONECUT2 binding site near the SMAD3 promoter under both normoxic and hypoxic conditions (Supplementary Figure 8a), and consistent with RNA-Seq data, silencing of ONECUT2 dramatically reduced SMAD3 RNA and protein abundance in PC3 cells as determined by RT-qPCR and western blot analyses (Fig. 3e). After confirming that SMAD3 was regulated by ONECUT2, we tested whether SMAD3 was required for HIF1a chromatin binding. In PC3 cells under hypoxic conditions, SMAD3 knockdown dramatically reduced HIF1a ChIP-Seq signal globally (Fig. 3f, Supplementary Figure 8b), and nearly $70 \%$ of the SMAD3 ChIP-Seq peaks overlapped with that of HIF1a (Fig. 3g).

To further pinpoint the core regulatory regions of SMAD3 and HIF1a, we performed SMAD3 and HIF1a ChIP-re-ChIP experiments followed by high-throughput sequencing (Supplementary Figure 9a). This analysis identified 12,445 highconfidence binding sites, of which $87 \%$ overlapped with both HIF1a and SMAD3 ChIP-Seq peaks (Fig. 3g). In contrast, SMAD3 and HIF2 $\alpha$ ChIP-re-ChIP-Seq identified only 324 peaks (2.7\%) overlapped with both HIF2 $\alpha$ and SMAD3 ChIP-Seq peaks (Supplementary Figure 9b), suggesting that SMAD3 directly interacts with HIF1a but not HIF2a. Among HIF1a ChIP-Seq peaks, the SMAD3-HIF1a core peaks (Fig. 3g, Group \#1), as exemplified by the ANGPTL4 and ADM locus (Fig. $3 \mathrm{~h}$, Supplementary Figure 9c), demonstrated strongest association with hypoxia-induced genes (Supplementary Figure 9d), further suggesting that ONECUT2-activated SMAD3 plays an important role in hypoxia signaling.

ONECUT2 and hypoxia synergize to drive neuroendocrine plasticity. Having demonstrated the function of ONECUT2 in NEPC, we further explored its role in prostate adenocarcinoma. Transient overexpression of ONECUT2 in LNCaP cells, an adeno-PCa cell line, was sufficient to induce the expression of NE marker genes including ASCL1, PEG10, and NSE (Fig. 4a, Supplementary Figure 10a), indicating that ONECUT2 is a regulator of NE plasticity.

Recent studies have reported that hypoxia can induce $\mathrm{NE}$ plasticity in $\mathrm{PCa}^{28,31,32}$. Consistent with these reports, LNCaP cells under hypoxic conditions demonstrated significantly higher levels of NE marker genes compared with the cells under normoxic conditions (Supplementary Figure 10b). To investigate whether there are synergies between ONECUT2 and hypoxia in driving NE plasticity, we evaluated the expression of NE marker genes under normoxic and hypoxic conditions with or without overexpression of ONECUT2. Interestingly, ONECUT2 overexpression and hypoxia synergized to drive elevated PEG10 
a

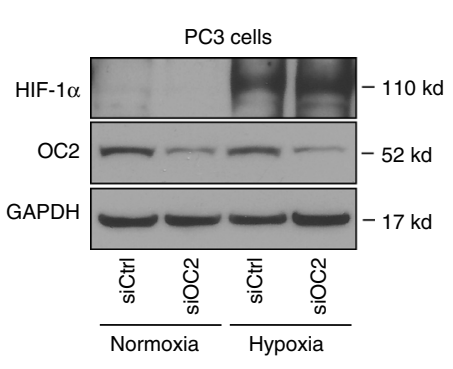

b

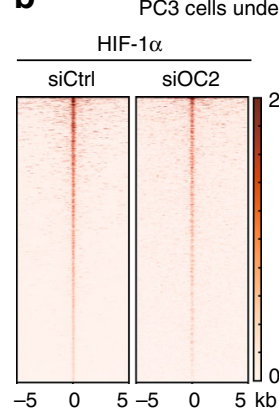

c

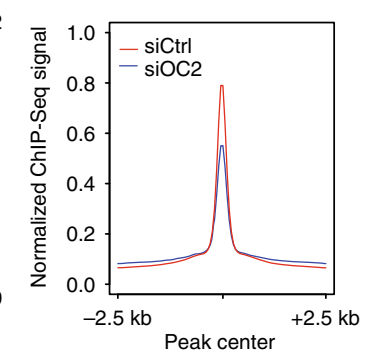

ANGPTL4

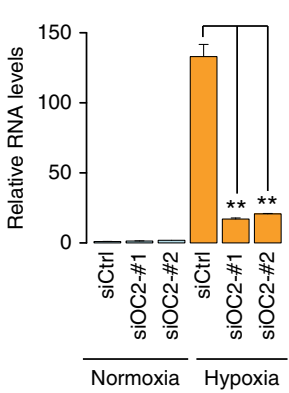

ADM

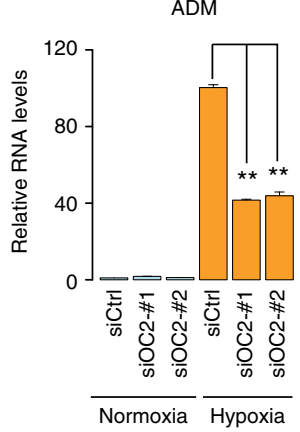

d

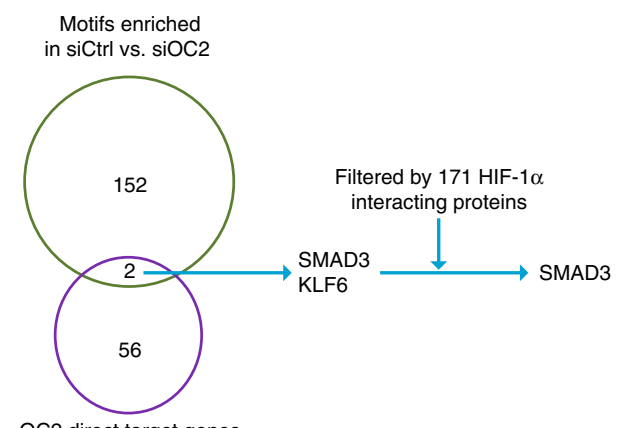

OC2 direct target genes

e

SMAD3
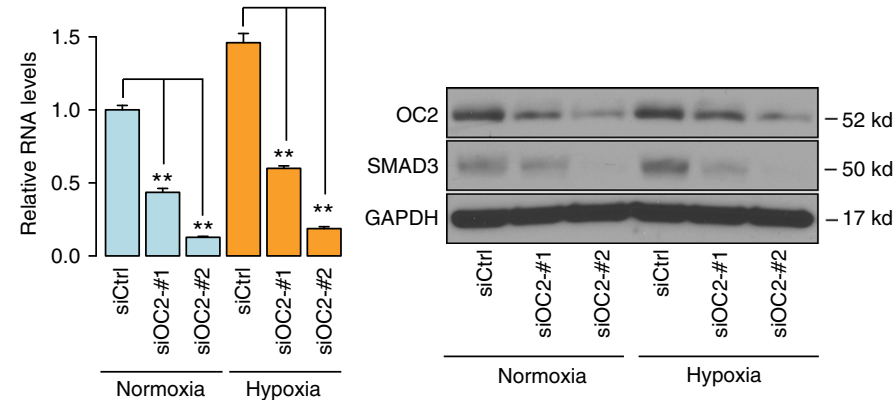

g
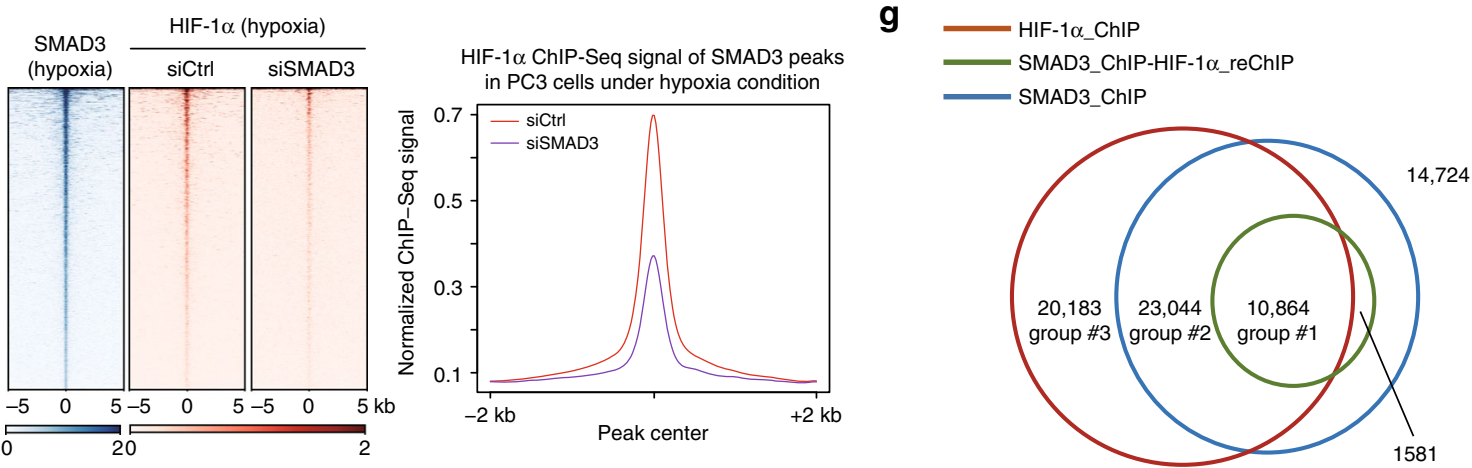

h

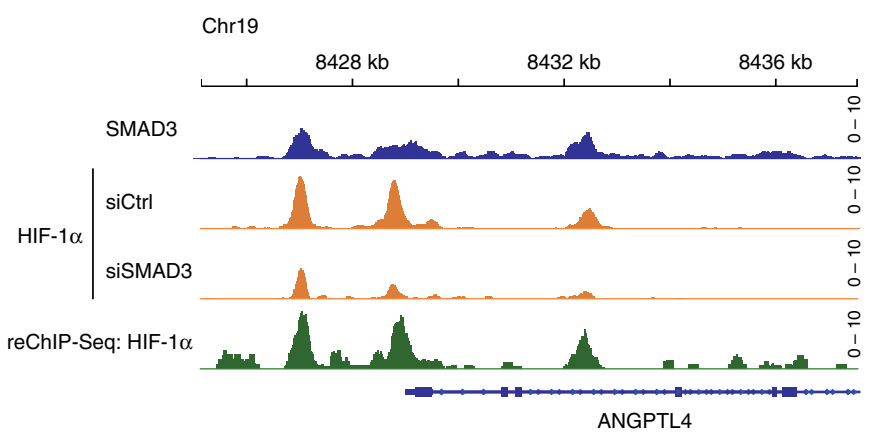

Chr22

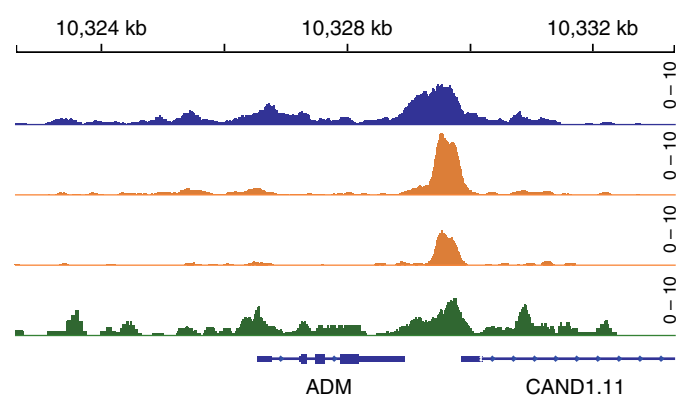

Fig. 3 ONECUT2 modulates HIF1 $\alpha$ binding to chromatin in NE-like PC3 cells. a Western blot of HIF1 $\alpha$ and ONECUT2 in PC3 cells under normoxic and hypoxic conditions. Two different siRNAs targeting ONECUT2 were mixed together for knockdown experiments. b Left panel: heatmaps show HIF1 $\alpha$ ChIPSeq signal in PC3 cells under hypoxic conditions with and without knockdown of ONECUT2; right panel: pileup of HIF1 $\alpha$ ChIP-Seq signals centered at HIF1 $\alpha$ ChIP-Seq peaks center. c Expression of ANGPTL4 and ADM, two hypoxia-regulated genes, with and without knockdown of ONECUT2 in PC3 cells. d Schematic illustration of the analysis identifying SMAD3 as an ONECUT2 regulated HIF1 $\alpha$ co-factor. Genes identified in motifs enriched in HIF1 $\alpha$ binding sites and ONECUT2 target genes were further filtered by HIF1 $\alpha$ interacting protein list from BioGRID. ONECUT2 target genes were defined as differentially expressed in ONECUT2 knockdown and control samples and with ONECUT2 binding sites nearby in PC3 cells under hypoxic conditions. e SMAD3 expression in response to ONECUT2 silencing in PC3 cells. $\mathbf{f}$ SMAD3 and HIF1 $\alpha$ binding sites with and without silencing of SMAD3. $\mathbf{g}$ The overlap of SMAD3 ChIP-Seq, HIF1 $\alpha$ ChIP-Seq and SMAD3-HIF1 $\alpha$ ChIP-re-ChIP-Seq peaks. h SMAD3 ChIP-Seq, HIF1 $\alpha$ ChIP-Seq and SMAD3-HIF1 $\alpha$ ChIP-re-ChIP-Seq signal near the promoter regions of hypoxia-induced genes ANGPTL4 and ADM. Error bars indicate s.d. from at least two technical replicates. $P$-value is calculated by one-way ANOVA. ${ }^{\star \star}: P<0.01$. Source data are provided as a Source Data file 
a

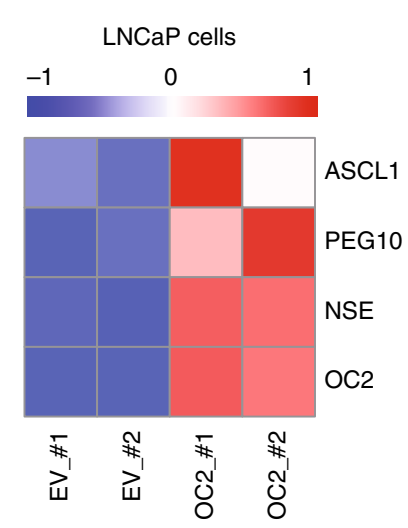

b

$\square$ EV PEG10 expression $\square$ OC2 in LNCaP cells

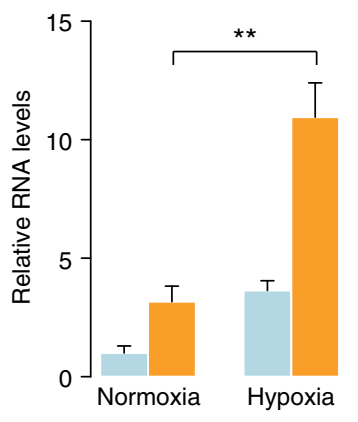

C

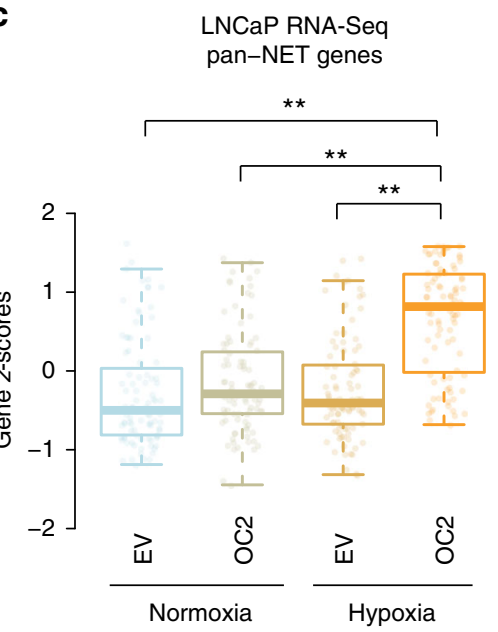

d

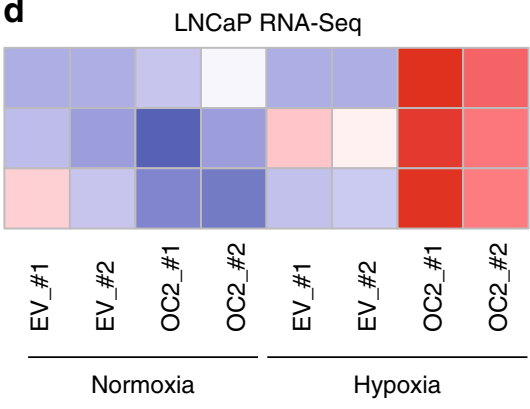

f

LNCaP RNA-Seq 243 DHT-induced genes

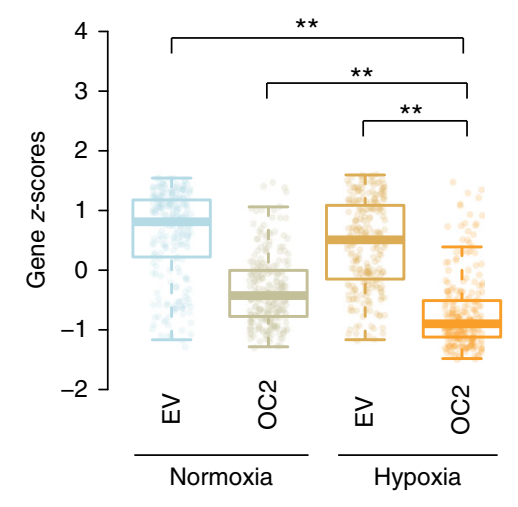

e
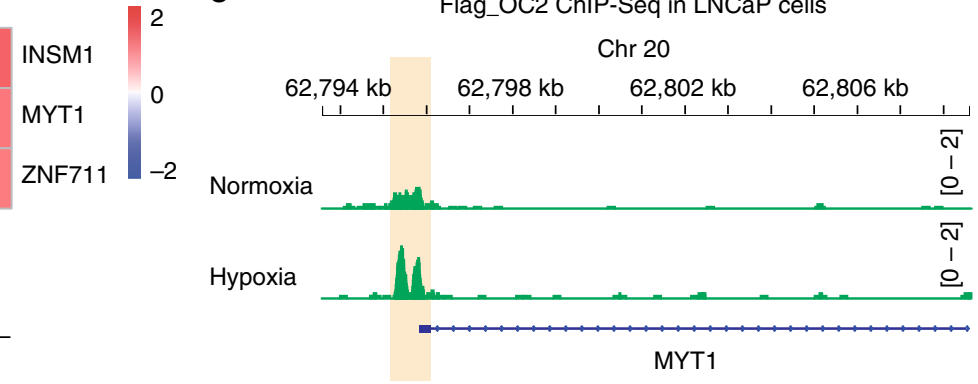

LNCaP cell neuritogenesis g

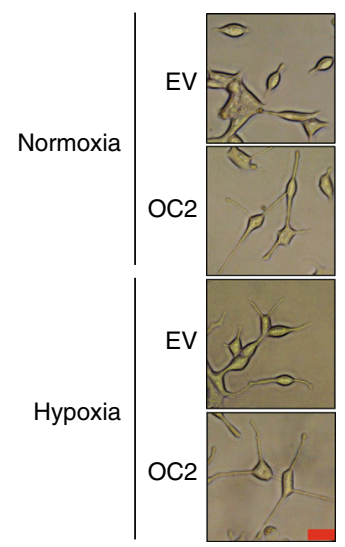

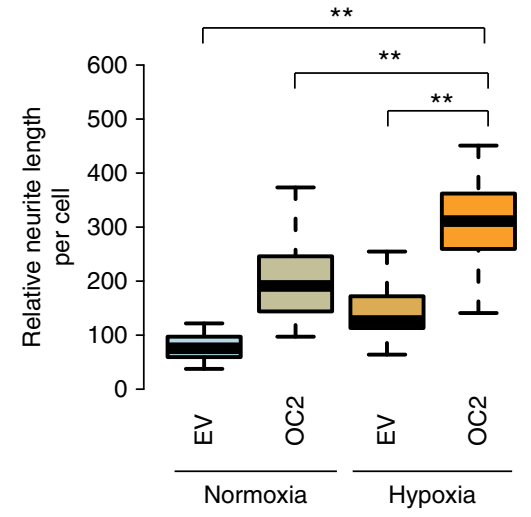

Fig. 4 ONECUT2 synergizes with hypoxia in driving neuroendocrine plasticity in prostatic adenocarcinoma. a NEPC marker gene expression in LNCaP cells. mRNA abundance as measured by RT-qPCR assay was first normalized by housekeeping gene RPS28, and then z-score normalized; red represents higher signal and blue represents lower signal. b Expression of PEG10 in ONECUT2 overexpressing LNCaP cells under normoxic and hypoxic conditions. $\mathbf{c}$ Zscores of the 92 pan-NET upregulated genes (ONECUT2 excluded) calculated in LNCaP RNA-Seq data. RNA-Seq experiments were performed in LNCaP cells with and without ONECUT2 overexpression under normoxic and hypoxic conditions. d Heatmap shows ONECUT2 overexpression and hypoxia synergistically promote expression of the core pan-NET transcriptional factors. e ONECUT2 ChIP-Seq signal at MYT1 promoter under normoxic and hypoxic conditions. $\mathbf{f}$ Z-scores of the 243 DHT-induced genes calculated in LNCaP RNA-Seq data. RNA-Seq experiments were performed in LNCaP cells with and without ONECUT2 overexpression under normoxic and hypoxic conditions. $\mathbf{g}$ Neuritogenesis analysis in LNCaP cell with and without overexpression of ONECUT2 under normoxic and hypoxic conditions. Error bars in $\mathbf{b}$ indicate s.d. from four technical replicates. $P$-values are calculated from a Student's $t$-test in $\mathbf{a}$ and $\mathbf{b}$ and Wilcoxon rank sum test in $\mathbf{c}$, $\mathbf{f}$, and $\mathbf{g}$. ${ }^{*}: P<0.05 .{ }^{\star *}: P<0.01$. EV empty vector control, OC2 ONECUT2 overexpression. Source data are provided as a Source Data file

mRNA abundance (Fig. 4b). To further explore the synergy between ONECUT2 and hypoxia in the development of NEPC, we performed RNA-Seq in LNCaP cells with or without ONECUT2 overexpression under normoxic and hypoxic conditions. Consistent with the results in PC3 cells, ONECUT2 overexpression in LNCaP cells promotes the expression of hypoxia hallmark genes under hypoxic conditions (Supplementary figure 10c-d). In addition, hypoxia treatment demonstrated strong synergy with ONECUT2 in activating expression of the 92 pan-NET upregulated genes (Fig. 4c; excluding ONECUT2). It is worth noting that three (i.e., MYT1, INSM1, and ZNF711) out of the four core pan-NET TFs (Fig. 1b, excluding ONECUT2) 
identified by our pan-NET analysis were synergistically upregulated by ONECUT2 overexpression and hypoxia treatment (Fig. 4d). Consistent with the mRNA abundance data, ONECUT2 bindings at the promoter of MYT1 and enhancers of INSM1 and ZNF711 were enhanced under hypoxic compared to normoxic conditions (Fig. 4e; Supplementary Figure 11a-b). In addition, ONECUT2 and hypoxia synergistically suppressed the expression of androgen-induced genes in LNCaP cells (Fig. 4f), consistent with the clinical observation of reduced AR signaling during NE transdifferentiation. Recent studies suggested that NE transdifferentiation from adeno-CRPC to NEPC is through an intermediary stem-like state, of which the cells exhibit epithelial-mesenchymal transition (EMT) and stem cell-like features ${ }^{25,33-35}$. ONECUT2 and hypoxia synergistically induced both EMT and stem cellrelated gene signatures in LNCaP cells (Supplementary Figure $11 \mathrm{c}-\mathrm{d})$, suggesting high lineage plasticity of those cells.

We next tested whether ONECUT2 and hypoxia collaborated to drive NE-like cell morphology in adeno-PCa cells. ONECUT2 overexpression or hypoxia treatment alone was able to induce neuritogenesis in LNCaP cells, and the combination has a much stronger effect (Fig. 4g). This observation was validated in adenoCRPC V16A cells (Supplementary Figure 12a-b). Knockdown of HIF $1 \alpha$ completely abolished ONECUT2 and hypoxia-induced NE marker genes expression (Supplementary Figure 12b), which indicated the essential role of HIF1 $\alpha$ in ONECUT2 and hypoxiainduced NE plasticity.

TP53 and RB1 are two most frequently mutated genes in NEPC, and TP53/RB1 double deletion has been reported to promote lineage plasticity of adeno-PCa ${ }^{25,33}$. We investigated whether ONECUT2 synergizes with TP53/RB1 double knockdown to further promote NE plasticity. In V16A cells, either ONECUT2 overexpression or TP53/RB1 double knockdown alone induced neuritogenesis and NE marker genes expression, but no strong additive effect was observed in combination of ONECUT2 overexpression and TP53/RB1 double knockdown (Supplementary Figure 12c-e). TP53/RB1 double knockdown or RB1 deletion resulted in increased expression of ONECUT2 in V16A and genetically engineered mouse models, respectively (Supplementary Figure $2 \mathrm{~d}$ and $12 \mathrm{e}$ ), suggesting the NE plasticity driven by TP53/RB1 deletion may partially be mediated through activation of ONECUT2.

NEPC with ectopic ONECUT2 expression is highly hypoxic. ONECUT2 overexpression synergizes with hypoxia to drive NE plasticity and aggressive tumor biology in NEPC. This therefore raises the question of whether NEPC is an inherently highly hypoxic tumor subtype. To address this question, we analyzed the expression of hypoxia marker gene CA9 by immunohistochemistry (IHC) in two independent tissue microarrays (TMAs) with 38 and 78 prostate tumor and benign tissue samples, respectively. In both TMAs, CA9 staining was significantly stronger in NEPC compared to primary adeno-PCa and adeno-CRPC (Fig. 5a-c), indicating that NEPC is more hypoxic than prostate adenocarcinoma. We further calculated hypoxia scores in two clinical cohorts of transcriptome data using a 32-gene hypoxia signature $^{36}$. In agreement with our tissue microarray analysis, NEPC samples exhibited significantly higher hypoxia scores compared to the adeno-CRPC samples (Supplementary Figure 13a). Consistently, in the RNA-Seq data of genetically engineered mouse model of PCa, DKO demonstrated the highest hypoxia score compared to SKO and WT (Supplementary Figure 13b).

To evaluate the relationship between ONECUT2 and prostate tumor hypoxia, we used the ONECUT2 up-regulated genes in PC3 cells under hypoxic condition as a signature of ONECUT2 activity. Only one gene was common between the hypoxia signature and ONECUT2 targets (Supplementary Figure 13c), excluding the potential bias caused by overlapped genes. ONECUT2 scores were positively correlated with hypoxia scores in primary prostate adenocarcinoma in two independent clinical cohorts, with $r=0.5$ and 0.6 respectively (Supplementary Figure 13d). This correlation slightly increased in adeno-CRPC samples $(r=0.627)$ and was highest in NEPC samples $(r=0.871)$ (Fig. 5d). These results, together with previous PIMO staining data in PC3 xenografts (Fig. 2f), indicate that ONECUT2 activity is increasingly correlated with $\mathrm{PCa}$ tumor hypoxia in the progression to NEPC.

Finally, having demonstrated that NEPC is more hypoxic than adeno-CRPC, we hypothesized that NEPC is more sensitive to hypoxia-directed treatment than adeno-CRPC. We therefore tested the tumor inhibition efficacy of a hypoxia-activated prodrug, TH-302 37 , in V16A and PC3 xenografts. TH-302 treatment dramatically reduced PC3 xenograft tumor growth (tumor inhibition rate $=84.7 \%$ ) but did not affect that of V16A xenograft (tumor inhibition rate $=1.6 \%$; Fig. $5 \mathrm{e}, \mathrm{g}$, left panels). Importantly, silencing of ONECUT2 in PC3 xenografts almost completely abolished the tumor inhibition effect of TH302, and overexpression of ONECUT2 sensitized the V16A xenografts to TH302 (Fig. 5e, f, right panels). In line with the tumor inhibition effects, PIMO staining in PC3 xenografts was much stronger than in V16A xenografts (Figs. 2f and 5f; Supplementary Figure 6a and 13e), and ONECUT2 knockdown reduced tumor hypoxia in PC3 xenografts, while ONECUT2 overexpression increased tumor hypoxia in V16A xenografts (Figs. 2f and 5f).

We further assessed the effect of $\mathrm{TH}-302$ on tumor growth of adeno-CRPC and NEPC patient-derived xenograft models. Weekly treatment of TH-302 dramatically inhibited NEPC PDX tumor growth in vivo compared to control group (tumor inhibition rate $=84.5 \%$ ), without obvious weight loss (Fig. 5h, i; Supplementary Figure 13f), but only had a moderate inhibitory effect on adeno-CRPC tumor growth (tumor inhibition rate $=$ 29.2\%, Fig. 5i). Furthermore, PIMO staining confirmed that $\mathrm{TH}-$ 302 treatment diminished tumor hypoxia in NEPC PDX tumors (Supplementary Figure 14). Altogether, these results suggest that hypoxia-directed therapy is a promising treatment option for patients with NEPC.

\section{Discussion}

While the mechanisms by which NEPC arises from prostate adenocarcinoma are poorly understood, recent studies suggest that transdifferentiation occurs from prostatic adenocarcinoma and not normal prostate glands ${ }^{10,38}$. In addition, t-NEPC cases, which can be induced from adeno-CRPC, are emerging with the widespread usage of potent AR-targeted agents in clinical practice $^{5}$. Therefore, alternative therapeutic options are becoming a pressing need for targeting the molecular mechanisms driving NEPC. To identify key drivers of NEPC, we conducted a panNET analysis by integrating four datasets comparing poorly differentiated NET vs. non-NET, including two of PCa. ONECUT2 was the top candidate regulator from our analysis.

In this study, we focused on PCa, and showed that ONECUT2 is upregulated in primary $\mathrm{PCa}$ compared with normal prostate tissues, and further upregulated in metastatic PCa. Similarly, higher ONECUT2 expression is also correlated with worse clinical outcomes in PCa patients. These observations indicate that ONECUT2 may play important roles in a broad spectrum of PCa progression. By using in vitro cell line models, we reveal that ONECUT2 regulates the aggressive tumor biology in NEPC, at least partially through activating SMAD3-HIF1a signaling. Finally, using cell line and patient-derived xenograft models, we provide evidence that a hypoxia-directed treatment potently 
a

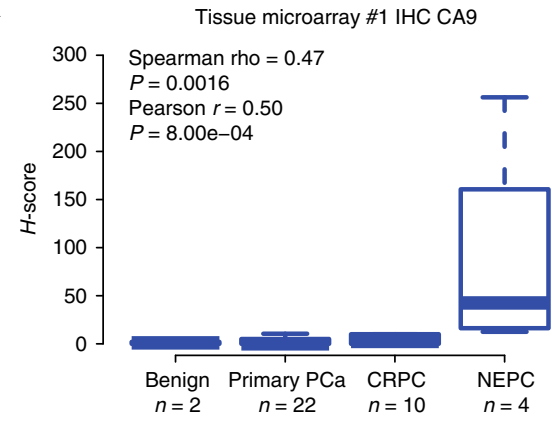

b

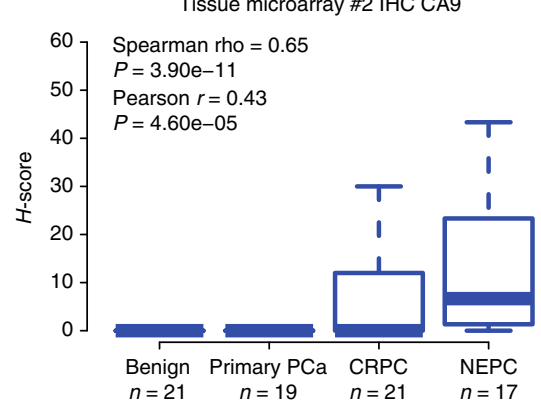

C

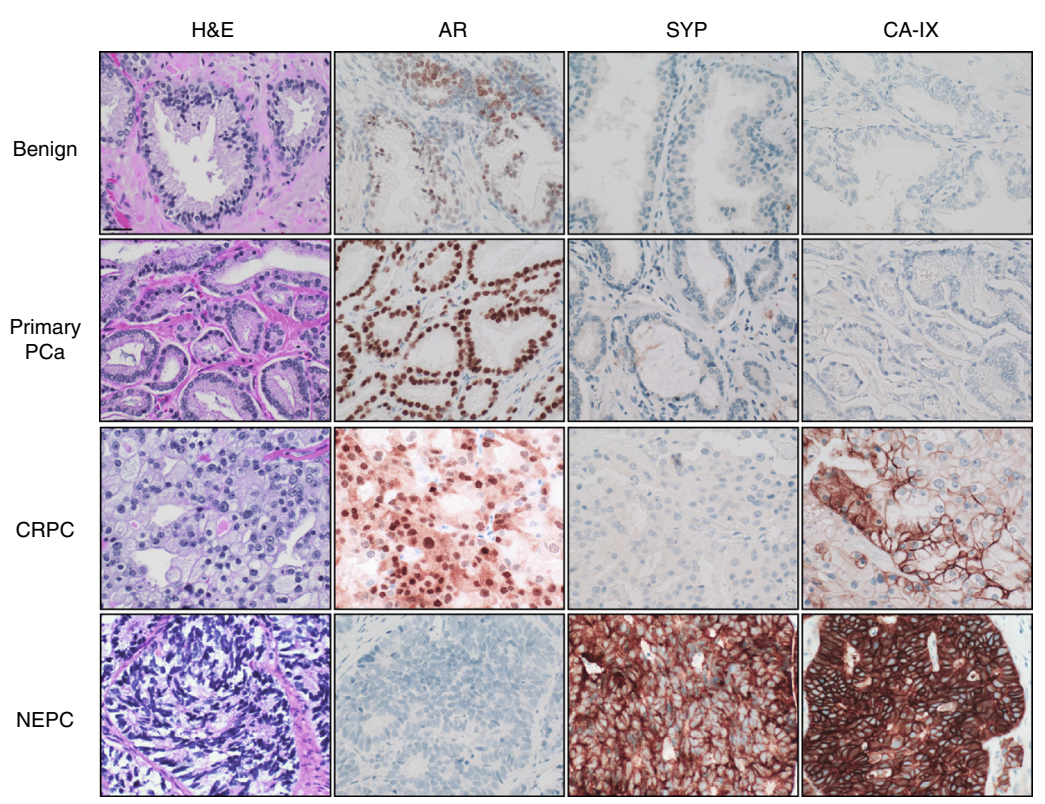

d

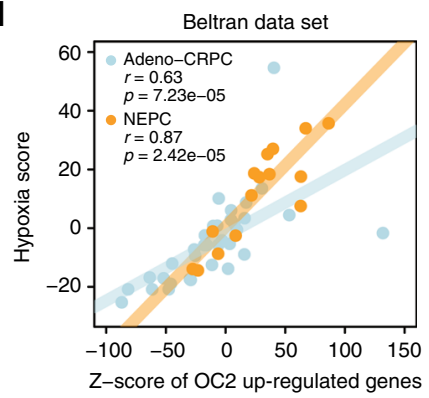

e

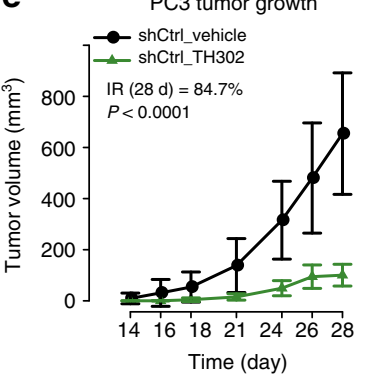

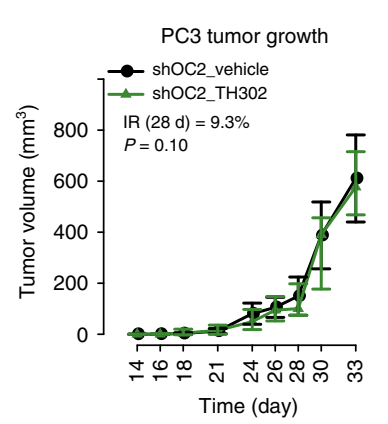

f

PIMO staining of V16A xenografts

EV

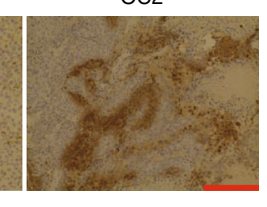

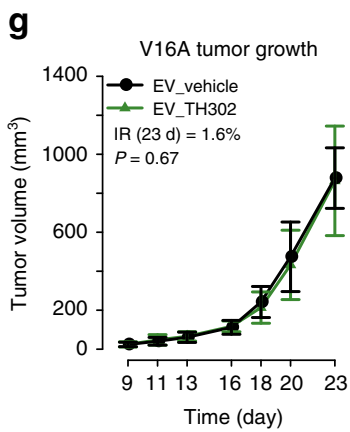
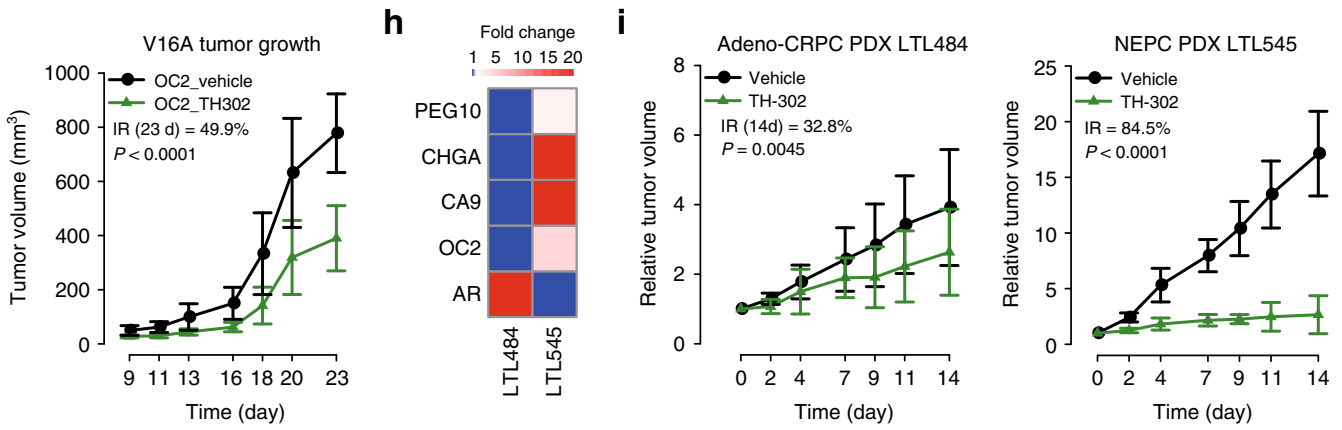

Fig. 5 NEPC is highly hypoxic and sensitive to hypoxia-directed treatment. a, b Hypoxia marker gene CA9 IHC staining in two independent tissue microarray sets. The combined $\mathrm{H}$-score, taking into account of both staining intensity and percentage, was used to quantify CA9 protein levels. (c) Representative images of tissue microarray analysis of AR, SYP, and CA9 IHC staining in benign prostate, primary PCa, adeno-CRPC, and NEPC tissues. Scale bar $=50 \mu \mathrm{m}$. $\mathbf{d}$ Correlation between ONECUT2 upregulated genes (summarized in z-scores) and hypoxia scores in Beltran adeno-CRPC/NEPC dataset. e PC3 xenograft tumor growth in response to hypoxia-activated prodrug TH-302 treatment, with and without silencing of ONECUT2. $n=7$ for shCtrl_Vehicle; $n=6$ for shCtrl_TH302; $n=8$ for shOC2_Vehicle; $n=7$ for shOC2_TH302. $\mathbf{f}$ Representative images of PIMO IHC staining of V16A xenograft tumors with and without overexpression of ONECUT2. Scale bar $=200 \mu \mathrm{m} . \mathbf{g}$ V16 A xenograft tumor growth in response to TH-302 with and without overexpression of ONECUT2. $n=7$ for EV_Vehicle; $n=8$ for EV_TH302; $n=6$ for OC2_Vehicle; $n=6$ for OC2_TH302. $\mathbf{h}$ Relative expression levels of related genes in the adeno-CRPC (LTL484) and NEPC (LTL545) PDX models determined by microarray analysis. i TH-302 treatment suppresses tumor growth in NEPC patient-derived xenografts. $n=4$ for vehicle group and $n=4$ for TH-302 group in NEPC PDXs; $n=5$ for vehicle group and $n=5$ for $\mathrm{TH}$-302 group in adeno-CRPC PDXs. In $\mathbf{e}, \mathbf{g}$, and $\mathbf{i}$, inhibition rate (IR) of tumor growth was calculated as (Tumor volume Vehicle - Tumor volume ${ }^{\mathrm{TH}-302}$ )/ Tumor volume Vehicle. $P$ values were determined by mixed-effects models of repeated-measures ANOVA for $\mathbf{e}, \mathbf{g}$, and $\mathbf{i}$. Source data are provided as a Source Data file 
inhibits NEPC tumor growth, and thus may serve as a promising treatment option for patients with NEPC.

We demonstrated that ONECUT2 synergizes with hypoxia to induce lineage plasticity towards a NE-like phenotype as measured by both morphological changes and NE marker genes expression in adeno-PCa cells. However, we acknowledge that our findings are limited by in vitro cell line models. Further validation in genetically engineered mouse or in pre-clinical PCa organoid models is warranted. The elevated ONECUT2 expression in adeno-CRPC tumors compared with primary PCa tumors also raises a possibility that some adeno-CRPC tumors may possess NE plasticity and molecular features and are at high risk for either progression or transition to NEPC. Blocking ONECUT2 and/or targeting hypoxia may prevent the development of NEPC for these patients at high risk and thus is worth exploring in future studies.

While we only validated the function of ONECUT2 in PCa, its function may not be limited to NEPC. Indeed, our analysis showed that ONECUT2 expression was elevated specifically, in poorly differentiated lung cancers and nervous system NETs compared with non-NETs. These data indicate that ONECUT2 may also have important function in poorly differentiated NETs of other cancer types besides NEPC. Therefore, the function of ONECUT2 in other types of NETs merits further investigation, especially in SCLC and poorly differentiated neuroblastoma. Additionally, ONECUT2 shows significant upregulation in cancers derived from multiple human tissues, indicating that ONECUT2 may play a critical role in tumorigenesis pan-cancer wide.

Besides ONECUT2, our analysis also identified eight other TFs upregulated in pan-NETs, including 4 TFs correlated with expression of the majority of other NET upregulated genes. Some of these TFs, such as ASCL1 and INSM1, have been well characterized in NET biology and survival ${ }^{17,39}$. However, for the remaining TFs, their functions have only been partially revealed in neuronal development or endocrine tissues, which are potentially related with NE differentiation/plasticity in tumors. Further studies of the function of these TFs may identify new drivers and therapeutic targets for poorly differentiated NETs.

Most solid tumors contain regions of hypoxia. The level of hypoxia varies in different tumor types and is a strong negative prognostic and predictive factor in multiple types of cancer, with particularly strong evidence in prostate cancer ${ }^{40,41}$. Selective tumor hypoxia targeting by hypoxia-activated pro-drug TH-302 in combination with immunotherapy is being evaluated in clinical studies in multiple cancer types, including locally advanced or metastatic PCa (ClinicalTrials.gov Identifier: NCT00743379). Our results showed that NEPC is more hypoxic than adeno-CRPC, and hypoxia targeting by $\mathrm{TH}-302$ dramatically reduced tumor growth of NEPC cell line and patient tumor derived xenografts. Furthermore, ONECUT2 is also expressed in certain normal tissues, such as brain, liver and pancreas, based on GTEx RNASeq data. Therefore, direct targeting of ONECUT2 may lead to unfavorable side effects, while targeting of ONECUT2-dependent tumor hypoxia could be a more convenient and optimal choice for NEPC patients. Altogether, our study demonstrated the importance of ONECUT2-hypoxia axis in the development of NEPC, and revealed hypoxia-directed treatment as a novel therapeutic option for NEPC patients.

\footnotetext{
Methods

Pan-cancer analysis. For pan-NET analysis, two PCa data sets generated by Beltran et al. and Lin et al. were used to identify genes up-regulated in NEPC compared with adeno-CRPC ${ }^{10,16}$. For the Lin dataset, one new NEPC PDX microarray data (LTL545) was added to increase statistical power. SCLC cell lines from CCLE (https://portals.broadinstitute.org/ccle/home) were used as NE type and NSCLC cell lines as non-NE type lung cancers. Neuroblastoma cell lines from
} CCLE were used as NE type and glioma as non-NE type nervous system tumors.
RNA-Seq data of corresponding cell lines were retrieved from CCLE to identify genes up-regulated in NE-type compared with non-NE type cancers. Wilcoxon test was used to calculate p-value in every comparison and Benjamini-Hochberg adjustment was conducted to assess the false discovery rates (FDR) of multiple comparisons. Genes co-up-regulated (fold change $>2$ and FDR $<0.05$ ) in NE vs. non-NE comparisons of all the four data sets were subjected to the following network analysis. For the network analysis, Pearson correlations were calculated between the co-up-regulated 9 TFs and 84 non-TF genes using their expression data in CCLE SCLC, CCEL neuroblastoma and Beltran NEPC data sets. Pearson correlations between the $10 \mathrm{TFs}$ were also added to the network. Correlation count was defined by $r>0.5$ in one dataset. Correlation count 1,2, or 3 indicates two genes are correlated $(r>0.5)$ in one, two or three data sets, respectively. Coexpression network was constructed based on the correlations and visualized by Gephi 0.9.1. Edge weights were defined by correlation counts. Node sizes were defined by weighted degrees. Communities in the network were detected by the modularity function ${ }^{42}$ in Gephi. Three NET vs. Non-NET data sets were used for further validation of ONECUT2 expression: (a) 29 SCLC RNA-Seq data from Rudin et al. dataset ${ }^{43}$ and 535 TCGA-LUAD RNA-Seq data from Genomic Data Commons Data Portal (https://portal.gdc.cancer.gov/); (b) 157 TARGET-NBL RNA-Seq data from Genomic Data Commons Data Portal and 529 TCGA-LGG RNA-Seq data from Genomic Data Commons Data Portal; (c) gene expression data during the transformation from prostatic adenocarcinoma (LTL331) to NEPC (LTL331R) from GSE59984 ${ }^{35}$. For pan-cancer tumor vs. normal analysis, TCGA level 3 RNA-Seq data were downloaded from Firehose Broad GDAC (https://gdac broadinstitute.org/, 2016-01-28 batch). For differential expression analysis, one cancer type was used only when the number of normal samples was more than 5 .

Cell culture and treatment. LNCaP, NCI-H660, and PC3 cell lines were obtained from the American Type Culture Collection (ATCC). V16A was established by Dr. Amina Zoubeidi's laboratory. All prostate cancer cell lines were cultured as recommended by ATCC. No mycoplasma contamination was detected in these cell lines using MycoAlert ${ }^{\mathrm{TM}}$ Mycoplasma Detection Kit (LT07-118, Lonza). PC3 cell proliferation was determined by AlamarBlue ${ }^{\text {ts }}$ (DAL1025, ThermoFisher) staining. PC3 cell migration and invasion were determined by transwell migration and matrigel invasion assays as described previously ${ }^{44}$. Briefly, PC3 cells were first transfected with ONECUT2 siRNAs and incubated for $48 \mathrm{~h}$. Then, $1 \times 10^{4}-1 \times 10^{5}$ cells were transferred to 24 -well transwell chambers (BD Biosciences, USA) followed by $24 \mathrm{~h}$ normoxia or hypoxia treatment. Cells that penetrated membrane were stained with crystal violet. For hypoxia treatment, cells were incubated in hypoxia chamber at $0.2 \% \mathrm{O}_{2}$. Fiji plugin Simple Neurite Track was used to quantify the neurite length of V16A and LNCaP cells following the developer's instruction $^{45}$.

siRNA transfection. siRNAs targeting ONECUT2, HIF1A, SMAD3, and control siRNAs were purchased from ThermoFisher. Lipofectamine RNAiMAX transfection reagent (13778150, ThermoFisher) was used for siRNA transfection following the manufacturer's instructions. The siRNA target sequences were listed in Supplementary Table 3 .

Vectors and lentiviral transfection. ONECUT2 overexpression vector pCMV6XL5-hONECUT2 was kindly provided by Dr. Merlin Crossley. To construct lentiviral vector of pLKO.1-TRC-shONECUT2, two shRNA oligonucleotides targeting different regions of ONECUT2 were inserted to pLKO.1-TRC vector (10878, Addgene), respectively. Sequences of ONECUT2 shRNAs are listed in Supplementary Table 3. To construct lentiviral vector of pLenti-CMV-Puro-DEST-FlagONECUT2, ONECUT2 protein coding DNA sequence was inserted to entry vector pENTR4-FLAG (17423, Addgene) first and then transferred to pLenti-CMV-PuroDEST (17452, Addgene). pLenti-C-Myc-DDK-P2A-Puro-ONECUT2

(RC211951L3) and pLenti-C-Myc-DDK-P2A-Puro (PS100092) were purchased from OriGene. Lentiviral particle production and infection were performed as described previously ${ }^{46}$. In brief, lentiviral vectors were co-transfected with psPAX2 and pMD2G vectors into HEK293T cells. Supernatants were collected at 24 and 48 $\mathrm{h}$ after transfection and stored in $-80^{\circ} \mathrm{C}$. For infection, $5 \times 10^{4}$ cells per well were seeded in six-well plates and infected with lentiviral supernatant on the following day.

Chromatin immunoprecipitation (ChIP) and ChIP-Seq. ChIP assay was performed using LNCaP and PC3 cells. Protein A (88845, ThermoFisher) and G (88847, ThermoFisher) Dynabeads were mixed at a 1:1 ratio, and pre-incubated with antibodies for 3 hrs before immunoprecipitation. LNCaP and PC3 cells were cross-linked by $1 \%$ formaldehyde for $10 \mathrm{~min}$ and then quenched with $125 \mathrm{mM}$ glycine. After a cold PBS wash, the nuclear fraction was extracted in $10 \mathrm{~mL}$ of LB1 buffer (50 mM HEPES-KOH, pH 7.5; 140 mM NaCl; 1 mM EDTA; 10\% Glycerol; $0.5 \%$ IGEPAL CA-630; $0.25 \%$ Triton X-100) for $10 \mathrm{~min}$ at $4{ }^{\circ} \mathrm{C}$. Nuclear fraction was then pelleted and resuspended in $10 \mathrm{~mL}$ of LB2 buffer $(10 \mathrm{mM}$ Tris-HCL, pH 8.0; $200 \mathrm{mM} \mathrm{NaCl} ; 1 \mathrm{mM}$ EDTA; $0.5 \mathrm{mM}$ EGTA) at $4{ }^{\circ} \mathrm{C}$ for 5 minutes. Nuclear fraction was pelleted again and resuspended in $\mathrm{LB} 3$ buffer $(10 \mathrm{mM}$ Tris- $\mathrm{HCl}, \mathrm{pH} 8$; $100 \mathrm{mM} \mathrm{NaCl} ; 1 \mathrm{mM}$ EDTA; 0.5 mM EGTA; $0.1 \% \mathrm{Na}$-Deoxycholate; $0.5 \% \mathrm{~N}$ lauroylsarcosine; Protease inhibitor cocktail). Nuclear fraction was then sonicated 
in a water bath sonicator (Diagenode bioruptor) to generate chromatin fragments at $\sim 300$ bp. $1 / 10$ volume of $10 \%$ Triton X-100 was added to chromatin lysate. Chromatin lysate was cleared by centrifugation and $1 / 10$ of supernatant was taken as input DNA. The rest chromatin lysate was divided equally to antibodyconjugated beads tubes and rotated at $4{ }^{\circ} \mathrm{C}$ overnight. Antibodies used for ChIP assays are anti-HIF1 $\alpha$ (NB100-479, Novus), anti-HIF2 $\alpha$ (NB100-122, Novus), antiSMAD3 (ab40854, Abcam) and anti-Flag (F3165, Sigma). The beads were washed in RIPA buffer (50 mM Tris, pH 7.6, $150 \mathrm{mM} \mathrm{NaCl}, 1 \mathrm{mM}$ EDTA, $0.1 \%$ SDS, $1 \%$ IGEPAL CA630, $0.5 \%$ sodium deoxycholate) and Elution buffer (0.1 M NaHCO3; $1 \%$ SDS; Proteinase K) was used to reverse cross-linking of DNA-protein complex at $65^{\circ} \mathrm{C}$ for $8-16 \mathrm{~h}$. DNA was purified by phenol-chloroform extraction and subjected to ChIP-Seq library preparation. For ChIP-Seq, $5 \mathrm{ng}$ of DNA (ChIPenriched or input) was used for library creation with the Rubicon ThruPLEX-FD kit and sequenced (75 bp single-end reads). All ChIP-Seq data were aligned using Bowtie2 (version 2.2.1) to the human genome of build version NCBI37/HG19. MACS2 was used for peak calling with the parameter "-SPMR" on ${ }^{47}$. Resultant bedgraph files were converted to big wiggle files by UCSC bedGraphToBigWig tool. The significantly enriched motifs in siCtrl HIF1 $\alpha$ ChIP-Seq peaks relative to siOC2 HIFla ChIP-Seq peaks were identified by CentriMo ${ }^{48}$

re-ChIP and re-ChIP-Seq. For re-ChIP assays, complexes from the primary immunoprecipitation were eluted from the beads by $37^{\circ} \mathrm{C}$ incubation in $20 \mathrm{mM}$ dithiothreitol for $30 \mathrm{~min}$. The eluates were then diluted at least 30 50 fold with dilution buffer ( $2 \mathrm{mM}$ EDTA, $150 \mathrm{mM} \mathrm{NaCl}, 1 \%$ Triton X-100, $20 \mathrm{mM}$ Tris- $\mathrm{HCl}$, $\mathrm{pH}$ 8.1) and subjected to a second immunoprecipitation reaction. Tris-EDTA buffer with 1\% SDS was used to elute re-ChIPed DNA. re-ChIP library preparation and sequencing were performed in the same way as ChIP-Seq. For re-ChIP-Seq analysis, after reads alignment, the primary ChIP-Seq bam file was used as the control for re-ChIP-Seq bam file in the peak calling by MACS2. To generate normalized bedgraph files of re-ChIP-Seq data, re-ChIP-Seq bedgraph file and primary ChIP-Seq bedgraph file were subjected to MASC2 bdgcmp sub-command with the option "-method = FE". Peak overlapping as shown by Venn diagram was determined by R package 'ChIPpeakAnno'49.

Western blotting. Western blotting was performed as described previously ${ }^{50}$. The blots were first incubated with anti-ONECUT2 (21916-1-AP, Proteintech; ab28466, Abcam), anti-SMAD3 (ab40854, Abcam), anti-HIF1a (NB100-479, Novus), antiH3 (ab1791, Abcam) or anti-GAPDH (ab1791, Abcam), and then with 1:10000 anti-rabbit secondary antibody (7074, Cell Signaling).

Mouse xenograft of PC3 cells and Patient-derived xenografts. All animal experiments were approved by the University Health Network Animal Care Committee (ID: AUP4714). Murine xenograft transplants were established directly from V16A cells with ONECUT2 overexpression or PC3 cells with ONECUT2 depletion using lentiviral shRNAs as described above. Briefly, $1 \times 10^{6} \mathrm{PC} 3$ or V16A cells resuspended in $100 \mu \mathrm{L}$ PBS/Matrigel (1:1) were subcutaneously injected into the upper right flank of male immunodeficient NOD-SCID mice between 5 and 8 weeks of age. Tumor growth was monitored by measuring two perpendicular diameters and calculating tumor volume $\left(\mathrm{mm}^{3}\right)$ using the formula $d^{2 *} D / 2$ where $d$ and $D$ are the shortest and longest diameter in $\mathrm{mm}$, respectively.

NEPC Patient-Derived Xenografts (PDX) LTL545 and adeno-CRPC PDX LTL484 were established in Dr. Wang lab ${ }^{16}$. These PDXs were propagated and maintained by subcutaneous passages in NOD-SCID mice. During all PDX implantation procedures, animals were anesthetized and their temperature was maintained at $38^{\circ} \mathrm{C}$ with a heating lamp. After PDX implantation, mice were randomized to vehicle or TH-302 treatment groups. After PDX tumor volumes reached $\sim 50 \mathrm{~mm}^{3}$. TH-302 was administered by intraperitoneal injection at $75 \mathrm{mg} / \mathrm{kg}$ once per week. Tumor growth monitor and volume calculation were performed as described above. To assess the effect of TH-302 treatment, PDX tumor volumes were normalized to the initial volumes at the beginning of TH-302 or vehicle treatment. To assess the treatment effects on tumor growth, tumor volume data at different time points were subjected to mixed-effects models for repeated-measures ANOVA by R package 'nlme'.

RNA-Seq. Total RNA of PC3 and LNCaP cells was extracted using RNeasy Mini Kit (74106, Qiagen) following manufacturer's procedure. Whole transcriptome sequencing libraries were prepared using TruSeq ${ }^{\oplus}$ Stranded mRNA Library Prep Kit (RS-122-2101, Illumina, San Diego, CA, USA) by following the manufacturer's instructions. RNA-Seq libraries were sequenced on a HiSeq2500 at Princess Margaret Genomic Centre. The trimmed reads were aligned to human genome hg19 with STAR (version 2.4.2a) ${ }^{51}$ and gene expression was then quantified using the reads per kilobase per million mapped reads (RPKM) method by Cufflinks (version 2.1.1) with GENCODE v24 GRCh37 GTF file. Gene Set Enrichment Analysis (GSEA) ${ }^{52}$ was used to evaluate the association of ONECUT2 expression with known pathways. GSEA was performed using the hallmark gene sets from version 4.0 of the molecular signature database (MSigDB) which are coherently expressed signatures derived by aggregating many MSigDB gene sets to represent well-defined biological states or processes.
Hypoxia score and ONECUT2-upregulated gene z-score. Hypoxia score and ONECUT2-upregulated gene $z$-score of each participant were defined as the sum of $z$-scores of corresponding signature genes. Hypoxia score is based on a 32-gene prostate cancer-specific hypoxia signature ${ }^{36}$. ONECUT2 score is based on the 123 ONECUT2-upregulated genes in PC3 cells under hypoxic conditions. For each gene, $z$-score $=(x-\mu) / \sigma ; x$ indicates pre-normalized gene expression level, $\mu$ indicates study mean of gene expression and $\sigma$ indicates study standard deviation of gene expression.

Immunohistochemistry. Preparation of paraffin-embedded tissue sections and immunohistochemical analyses of PC3 and V16A xenografts and NEPC PDXs were carried out at histology core facility of Princess Margaret Cancer Centre. Paraffin sections at $4 \mu \mathrm{m}$ thickness were dried at $60^{\circ} \mathrm{C}$ oven for 2 hours before staining. The immunohistochemistry (IHC) was performed according to the manufacture's guidelines using BenchMark XT-an automated slide strainer (Ventana Medical System) with standard antigen retrieval (CC1, pH 8.0, \#950124). For PIMO staining, $60 \mathrm{mg} / \mathrm{kg}$ PIMO HCl (Hypoxyprobe ${ }^{\mathrm{mt}}-1$, Hypoxyprobe) was intraperitoneal injected into tumor bearing mice and tumors were collected 60 minutes later. Mouse monoclonal anti-PIMO antibody (MAb1, Hypoxyprobe, 1:400 dilution), rabbit polyclonal anti-CA9 antibody (NB100-417, Novus, 1:1000 dilution), rabbit polyclonal anti-SYP antibody (ab32127, Abcam, 1:1000 dilution) and rabbit polyclonal anti-CHGA antibody (ab15160, Abcam, 1:1000 dilution) were used for immunohistochemistry. Biotinylated anti-rabbit IgG (Vector, BA-1000) was added to slides at 1:200 for 12 minutes. The primarysecondary antibody complex was then visualized with Ventana iView DAB Detection Kit (\#760-091). The slides were counterstained with Harris hematoxylin, dehydrated in graded alcohol, cleared in xylene and coverslipped in Permount.

Tissue microarray. In this study, we conducted two independent tissue microarray analysis using TMAs from the Vancouver Prostate Cancer Tissue Bank (TMA \#1) and the Weill Cornell Medicine (TMA \#2).

TMA \# 1 consists a total of 38 prostate tumor and benign tissue samples, which were obtained from the Vancouver Prostate Centre Tissue Bank. H\&E slides were first reviewed and desired areas were marked also on their correspondent paraffin blocks. A TMA was created by taking double $1 \mathrm{~mm}$ cores from matching selected areas of paraffin blocks using a semi-automated tissue microarrayer from Pathology Devices TMA arrayer with Leica M50 stereomicroscope. Immunohistochemical staining was conducted by Ventanaautostainer model Discover XT ${ }^{\mathrm{px}}$ (Ventana Medical System, Tuscan, Arizona) with enzyme labeled biotin streptavidin system and solvent-resistant DAB Map kit by using 1/200 concentration of CA9 mouse monoclonal antibody. All stained slides were digitalized with the SL801 autoloader and Leica SCN400 scanning system (Leica Microsystems; Concord, Ontario, Canada) at magnification equivalent to $\times 40$. The images were subsequently stored in the SlidePath digital imaging hub (DIH; Leica Microsystems) of the Vancouver Prostate Centre. Prostate cancer specimens were obtained from patients following a protocol approved by the Clinical Research Ethics Board of the University of British Columbia and the BC Cancer Agency (all patients signed a consent form approved by the Ethics Board).

For TMA \#2, benign prostate $(n=21)$, prostate adenocarcinoma $(n=19)$, castration-resistant prostate adenocarcinoma (CRPC) $(n=21)$ and neuroendocrine prostate cancer (NEPC) $(n=17)$ samples were evaluated and scored at WCM for expression of androgen receptor (AR), synaptophysin (SYP), and CA9. Antibodies used were: CA9 (Novus Biologicals \#NB100-417, Citratebased antigen retrieval, 1:1000) AR (Biogenex, \#MU256-UC, EDTA-based antigen retrieval, 1:800), SYP (Leica PA0299, EDTA-based antigen retrieval). Prostate cancer specimens were obtained from patients following a protocol approved by the Weill Cornell Medicine (WCM) Institutional Review Board (IRB) with informed consent.

Each core of the TMA was scored as $\%$ of cells with $0=$ no expression, $1=$ low expression, 2 = moderate expression, and 3 = high expression of CA9. H-score analysis was based on $\%$ of positive cells and intensity of staining in positive cells (range 0-300). Images where taken at $\times 40$, scales bar $50 \mu \mathrm{m}$.

\section{Data availability}

The RNA-seq and ChIP-seq raw sequence tags and processed bed files reported in this manuscript have been submitted to the National Centre for Biotechnology Gene Expression Omnibus (GEO) database (accession no. GSE106305). The data that support the findings of this study are available from the corresponding author upon reasonable request. A reporting summary for this Article is available as a Supplementary Information file. The source data underlying Figs. 2g, h, 3a, c, e, 4b, and 5e, g, h and Supplementary Figs 4a, 4b, 4c, 4f, 6c, 7a, 9c, 10a, 11b and 11e are provided in Supplementary Data 1.

Received: 2 August 2018 Accepted: 17 December 2018 Published online: 17 January 2019 


\section{References}

1. Rickman, D. S., Beltran, H., Demichelis, F. \& Rubin, M. A. Biology and evolution of poorly differentiated neuroendocrine tumors. Nat. Med. 23, 1-10 (2017).

2. Aggarwal, R., Zhang, T., Small, E. J. \& Armstrong, A. J. Neuroendocrine prostate cancer: subtypes, biology, and clinical outcomes. J. Natl. Compr. Cancer Netw. 12, 719-726 (2014).

3. Vashchenko, N. \& Abrahamsson, P. A. Neuroendocrine differentiation in prostate cancer: implications for new treatment modalities. Eur. Urol. 47, 147-155 (2005).

4. Beltran, $\mathrm{H}$. et al. Challenges in recognizing treatment-related neuroendocrine prostate cancer. J. Clin. Oncol. 30, e386-e389 (2012).

5. Bluemn, E. G. et al. Androgen receptor pathway-independent prostate cancer is sustained through FGF signaling. Cancer Cell 32, 474-489 e476 (2017).

6. Aggarwal A. et al. Clinical and genomic characterization of treatmentemergent small-cell neuroendocrine prostate cancer: a multi-institutional prospective study. J. Clin. Oncol. 36, 2492-2503 (2018).

7. Aparicio, A. M. et al. Platinum-based chemotherapy for variant castrateresistant prostate cancer. Clin. Cancer Res. 19, 3621-3630 (2013).

8. Papandreou, C. N. et al. Results of a phase II study with doxorubicin etoposide, and cisplatin in patients with fully characterized small-cell carcinoma of the prostate. J. Clin. Oncol. 20, 3072-3080 (2002).

9. George, J. et al. Comprehensive genomic profiles of small cell lung cancer. Nature 524, 47-53 (2015).

10. Beltran, H. et al. Divergent clonal evolution of castration-resistant neuroendocrine prostate cancer. Nat. Med. 22, 298-305 (2016).

11. Oberg, K. et al. Consensus on biomarkers for neuroendocrine tumour disease. Lancet Oncol. 16, e435-e446 (2015).

12. Ferolla, $\mathrm{P}$. et al. The biological characterization of neuroendocrine tumors: the role of neuroendocrine markers. J. Endocrinol. Invest. 31, 277-286 (2008)

13. Vinik, A. I. et al. NANETS consensus guidelines for the diagnosis of neuroendocrine tumor. Pancreas 39, 713-734 (2010).

14. Niederle, M. B., Hackl, M., Kaserer, K. \& Niederle, B. Gastroenteropancreatic neuroendocrine tumours: the current incidence and staging based on the WHO and European Neuroendocrine Tumour Society classification: an analysis based on prospectively collected parameters. Endocr. Relat. Cancer 17, 909-918 (2010).

15. Eads, J. R. Poorly differentiated neuroendocrine tumors. Hematol. Oncol. Clin. North. Am. 30, 151-162 (2016).

16. Lin, D. et al. High fidelity patient-derived xenografts for accelerating prostate cancer discovery and drug development. Cancer Res. 74, 1272-1283 (2014).

17. Augustyn, A. et al. ASCL1 is a lineage oncogene providing therapeutic targets for high-grade neuroendocrine lung cancers. Proc. Natl Acad. Sci. USA 111, 14788-14793 (2014)

18. Rosenbaum, J. N. et al. INSM1: a novel immunohistochemical and molecular marker for neuroendocrine and neuroepithelial neoplasms. Am. J. Clin. Pathol. 144, 579-591 (2015).

19. Jernman, J. et al. PROX1 is involved in progression of rectal neuroendocrine tumors, NETs. Virchows Arch. 467, 279-284 (2015).

20. Arda, H. E. et al. Age-dependent pancreatic gene regulation reveals mechanisms governing human beta cell function. Cell. Metab. 23, 909-920 (2016).

21. Vasconcelos, F. F. et al. MyT1 counteracts the neural progenitor program to promote vertebrate neurogenesis. Cell Rep. 17, 469-483 (2016).

22. Mall, M. et al. Myt1l safeguards neuronal identity by actively repressing many non-neuronal fates. Nature 544, 245-249 (2017).

23. Sapkota, D. et al. Onecut 1 and Onecut 2 redundantly regulate early retinal cell fates during development. Proc. Natl Acad. Sci. USA 111, E4086-E4095 (2014).

24. Guo, H. et al. Modulation of long noncoding RNAs by risk SNPs underlying genetic predispositions to prostate cancer. Nat. Genet. 48, 1142-1150 (2016).

25. $\mathrm{Ku}, \mathrm{S}$. Y. et al. Rb1 and Trp53 cooperate to suppress prostate cancer lineage plasticity, metastasis, and antiandrogen resistance. Science 355, 78-83 (2017).

26. Tai, S. et al. PC3 is a cell line characteristic of prostatic small cell carcinoma. Prostate 71, 1668-1679 (2011)

27. Milosevic, M. et al. Tumor hypoxia predicts biochemical failure following radiotherapy for clinically localized prostate cancer. Clin. Cancer Res. 18, 2108-2114 (2012)

28. Qi, J. et al. Siah2-dependent concerted activity of HIF and FoxA2 regulates formation of neuroendocrine phenotype and neuroendocrine prostate tumors. Cancer Cell 18, 23-38 (2010).

29. Dengler, V. L., Galbraith, M. \& Espinosa, J. M. Transcriptional regulation by hypoxia inducible factors. Crit. Rev. Biochem. Mol. Biol. 49, 1-15 (2014).

30. Semenza, G. L. Hypoxia-inducible factors in physiology and medicine. Cell 148, 399-408 (2012).

31. Danza, G. et al. Notch signaling modulates hypoxia-induced neuroendocrine differentiation of human prostate cancer cells. Mol. Cancer Res. 10, 230-238 (2012).
32. Lin, T. P. et al. REST reduction is essential for hypoxia-induced neuroendocrine differentiation of prostate cancer cells by activating autophagy signaling. Oncotarget 7, 26137-26151 (2016).

33. $\mathrm{Mu}, \mathrm{P}$. et al. SOX 2 promotes lineage plasticity and antiandrogen resistance in TP53- and RB1-deficient prostate cancer. Science 355, 84-88 (2017).

34. Nouri, M. et al. Therapy-induced developmental reprogramming of prostate cancer cells and acquired therapy resistance. Oncotarget 8, 18949-18967 (2017)

35. Akamatsu, S. et al. The placental gene PEG10 promotes progression of neuroendocrine prostate cancer. Cell Rep. 12, 922-936 (2015).

36. Ragnum, H. B. et al. The tumour hypoxia marker pimonidazole reflects a transcriptional programme associated with aggressive prostate cancer. $\mathrm{Br}$. J. Cancer 112, 382-390 (2015)

37. Duan, J. X. et al. Potent and highly selective hypoxia-activated achiral phosphoramidate mustards as anticancer drugs. J. Med. Chem. 51, 2412-2420 (2008).

38. Zou, M. et al. Transdifferentiation as a mechanism of treatment resistance in a mouse model of castration-resistant prostate cancer. Cancer Discov. 7, 736-749 (2017)

39. Fujino, K. et al. Insulinoma-associated protein 1 is a crucial regulator of neuroendocrine differentiation in lung cancer. Am. J. Pathol. 185, 3164-3177 (2015).

40. Lalonde, E. et al. Tumour genomic and microenvironmental heterogeneity for integrated prediction of 5-year biochemical recurrence of prostate cancer: a retrospective cohort study. Lancet Oncol. 15, 1521-1532 (2014).

41. Chua, M. L. K. et al. A prostate cancer "Nimbosus": genomic instability and SChLAP1 dysregulation underpin aggression of intraductal and cribriform subpathologies. Eur. Urol. 72, 665-674 (2017)

42. Subelj, L. \& Bajec, M. Unfolding communities in large complex networks: combining defensive and offensive label propagation for core extraction. Phys Rev. E. Stat. Nonlin. Soft. Matter Phys. 83, 036103 (2011). (3 Pt 2).

43. Rudin, C. M. et al. Comprehensive genomic analysis identifies SOX2 as a frequently amplified gene in small-cell lung cancer. Nat. Genet. 44, 1111-1116 (2012).

44. Li, Y. et al. Spliceosome-associated factor CTNNBL1 promotes proliferation and invasion in ovarian cancer. Exp. Cell Res. 357, 124-134 (2017).

45. Longair, M. H., Baker, D. A. \& Armstrong, J. D. Simple Neurite Tracer: open source software for reconstruction, visualization and analysis of neuronal processes. Bioinformatics 27, 2453-2454 (2011).

46. Guo, H. et al. Chemokine receptor CXCR2 is transactivated by $\mathrm{p} 53$ and induces p38-mediated cellular senescence in response to DNA damage. Aging Cell. 12, 1110-1121 (2013).

47. Zhang, Y. et al. Model-based analysis of ChIP-Seq (MACS). Genome Biol. 9, R137 (2008)

48. Bailey, T. L. \& Machanick, P. Inferring direct DNA binding from ChIP-seq. Nucleic Acids Res. 40, e128 (2012).

49. Zhu, L. J. et al. ChIPpeakAnno: a Bioconductor package to annotate ChIP-sec and ChIP-chip data. BMC Bioinforma. 11, 237 (2010).

50. Wei, Z. et al. CUL4B impedes stress-induced cellular senescence by dampening a p53-reactive oxygen species positive feedback loop. Free Radic Biol. Med. 79, 1-13 (2015).

51. Dobin, A. et al. STAR: ultrafast universal RNA-seq aligner. Bioinformatics 29 , 15-21 (2013).

52. Subramanian, A. et al. Gene set enrichment analysis: a knowledge-based approach for interpreting genome-wide expression profiles. Proc. Natl Acad. Sci. USA 102, 15545-15550 (2005).

\section{Acknowledgements}

The authors thank Dr. Merlin Crossley for sharing of ONECUT2 overexpression plasmids, Juan Miguel Mosquera for reviewing the TMAs and the Princess Margaret Genomic Centre for high-throughput sequencing support. The work was supported by Princess Margaret Cancer Foundation (to H.H.H.), Canada Foundation for Innovation and Ontario Research Fund (CFI32372 to H.H.H.), NSERC discovery grant (498706 to H.H.H.), WICC Ontario 20th Anniversary Prostate Cancer Innovation Grant of the CCS (703800 to H.H.H.), CIHR operating grant (142246, 152863, 152864 and 159567 to H.H H. and 153081 to Y.Z.W.), Movember Rising Star awards from Prostate Cancer Canada (RS2014-01 to P.C.B. and RS2016-1022 to H.H.H.), TFRI program project (1062 to Y.Z. W.) and NIH/NCI SPORE in Prostate Cancer (P50-CA211024 to H.B.). H.H.H. holds a TFRI New Investigator Award, a CIHR New Investigator Award and an OMIR Early Researcher Award. J.H. is a CIHR Graduate Student Fellowship recipient. P.C.B. was supported by a TFRI New Investigator Award and a CIHR New Investigator Award. The results shown here are in whole or part based upon data generated by the TCGA Research Network.

\section{Author contributions}

H.G., X.C., Y.W., and H.H.H. designed the studies and wrote the manuscript; H.G., X.C., J.H. and F.S. performed the experiments with the help from D.L., L.P., E.L., P.S., T.N., Y. 
L., Y.Z., X.X., J.X., S.Z., O.M., A.V., H.X. and R.N.V.; M.A., H.G., and H.H.H. conducted the data analysis with the help from S.C., A.V.S., W.B.A., M.K., M.G., R.G.B., J.T.P., C.R., B.H.K., M.S.T., B.G.W., F.F., L.F., A.B., L.E., T.v.d.K., P.C.B., A.Z. and H.B.; M.G., R.G.B., B.H.K., M.S.T., F.F., L.F., A.Z., Y.W. and P.C.B. revised the manuscript.

\section{Additional information}

Supplementary Information accompanies this paper at https://doi.org/10.1038/s41467018-08133-6.

Competing interests: The authors declare no competing interests.

Reprints and permission information is available online at http://npg.nature.com/ reprintsandpermissions/.

Journal peer review information: Nature Communications thanks the anonymous reviewers for their contribution to the peer review of this work.
Publisher's note: Springer Nature remains neutral with regard to jurisdictional claims in published maps and institutional affiliations.

(c) Open Access This article is licensed under a Creative Commons Attribution 4.0 International License, which permits use, sharing, adaptation, distribution and reproduction in any medium or format, as long as you give appropriate credit to the original author(s) and the source, provide a link to the Creative Commons license, and indicate if changes were made. The images or other third party material in this article are included in the article's Creative Commons license, unless indicated otherwise in a credit line to the material. If material is not included in the article's Creative Commons license and your intended use is not permitted by statutory regulation or exceeds the permitted use, you will need to obtain permission directly from the copyright holder. To view a copy of this license, visit http://creativecommons.org/licenses/by/4.0/.

(C) The Author(s) 2019 